\title{
Timing and location of substorm onsets from THEMIS satellite and ground based observations
}

\author{
S. Mende ${ }^{1}$, V. Angelopoulos ${ }^{2}$, H. U. Frey ${ }^{1}$, E. Donovan ${ }^{3}$, B. Jackel ${ }^{3}$, K.-H. Glassmeier ${ }^{4}$, J. P. McFadden ${ }^{1}$, D. Larson ${ }^{1}$, \\ and C. W. Carlson ${ }^{1}$ \\ ${ }^{1}$ Space Sciences Laboratory, University of California, Berkeley, CA, USA \\ ${ }^{2}$ IGPP, ESS, University of California, Los Angeles, CA, USA \\ ${ }^{3}$ University of Calgary, Calgary, Alberta, Canada \\ ${ }^{4}$ Institute for Geophysics and extraterrestrial Physics, TU Braunschweig, Germany
}

Received: 28 October 2008 - Revised: 20 May 2009 - Accepted: 23 June 2009 - Published: 15 July 2009

\begin{abstract}
The unprecedented coverage of the THEMIS GBO station network coupled with high temporal and spatial resolution allowed us to determine the various stages of the global scale developments of the optical aurora at substorm onsets. We identified several steps of the substorm onset auroral phenomena and we suggest that the most rapid development is the starting of the Substorm Poleward Expansion (SPE) and it is most useful for accurate timing of the substorm onset. The physical significance of this step is the start of the large scale substorm energy dissipation in the atmosphere due to particle precipitation and auroral electrojet currents. We also recognized several pre-cursor features. We also measured the time of arrival of magnetic impulses associated with the same substorms at the THEMIS satellites. We used these times and a simple model with assumed iono-acoustic speeds in the range of $300-800 \mathrm{~km} / \mathrm{s}$ to calculate the location and time of the origin of the magnetic impulses propagating from substorm onset. The assumption was made that the substorm occurred between two THEMIS satellites and the impulses propagated away from a singular starting point in and out along the magneto tail GSM-x axis. This technique is only useful in cases where the ground based signature of the substorm is very close in local time (or longitude) to the foot of the field lines of the THEMIS satellites. The $\mathrm{x}$ distance of the calculated origins were naturally highly dependent on the assumed propagation velocity model and the associated magneto-sonic speed. The resulting $\mathrm{x}$ distances of the starting point for the three events ranged between 11 and $17.6 R_{E}$. denoting a starting region that requires highly stretched field lines to map to the auroral onset latitude but which is generally considered to be too close for
\end{abstract}

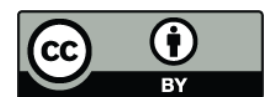

Correspondence to: S. Mende (mende@ssl.berkeley.edu) neutral line formation. The corresponding start times were in the range of 0 to $170 \mathrm{~s}$ prior SPE depending strongly on the assumed propagation speed.

Keywords. Magnetospheric physics (Auroral phenomena; Magnetospheric configuration and dynamics; Storms and substorms)

\section{Introduction}

Substorms are large scale magnetospheric instabilities during which magnetic energy is rapidly converted into particle energy and some of the energy dissipated in spectacular auroral displays. Our understanding of how the magnetosphere works is incomplete without understanding what triggers and drives substorms. Since their early description e.g. Akasofu (1964) a great deal of effort has been expanded in studying substorms but we are still unable to settle fundamental controversies about what drives substorms, where they start in the magnetosphere and which direction they propagate along the Earth magnetic tail. According to most current substorm models the substorm process initiates either with a decrease of cross tail current in the inner plasma sheet due to some kind of instability at $<10 R_{E}$ (Roux et al., 1991; Lui et al., 1991) with an outward propagation of the phenomena along the tail, or with the initiation point located at the tail reconnection region at $>20 R_{E}$ (Baker et al., 1996) with an inward propagation of the phenomena towards the earth while the initiation preceding the auroral signature visible from the ground. The NASA program entitled "Time History of Events and Macroscale Interactions during Substorms" (THEMIS) (Angelopoulos, 2008a) provides fundamentally new opportunities to resolve this problem. In this program five identical probes (satellites) were placed on special orbits

Published by Copernicus Publications on behalf of the European Geosciences Union. 


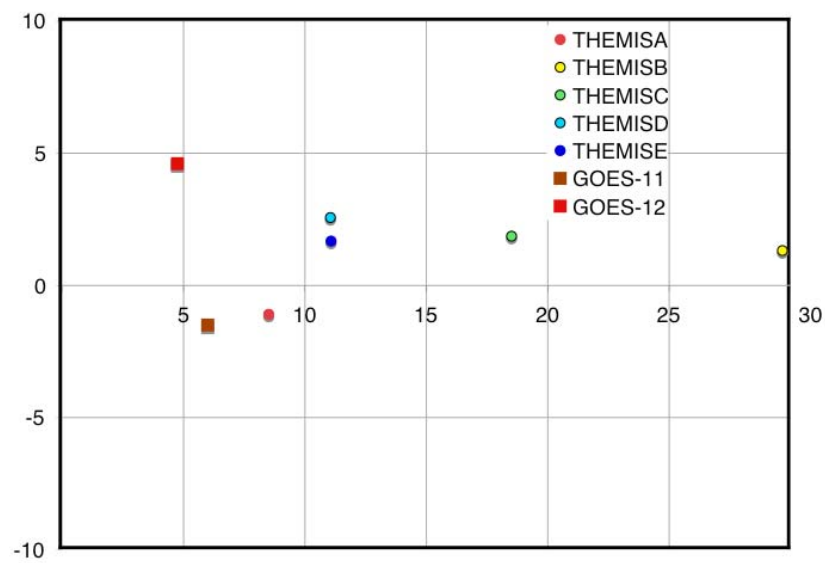

Fig. 1. THEMIS satellite positions GSM x and y in units of $R_{E}$ for 2 February 2008 at 08:00 UT.

which permit the alignment of the satellites in the tail and the instruments on the satellite measure the in situ particles and fields at their location. Correlative measurements from five strategically located identical probes can observe substorm associated magnetic field pulses and from their timing, the direction of propagation can be infered. In rarer cases where the satellites are located inside the substorm associated particle streams they can directly measure the propagation velocities. It is therefore a main goal of THEMIS to locate the point and time of origin in the magnetosphere and compare it to the auroral signature at substorm onset. The THEMIS satellite constellation consists of three inner probes monitoring the region at $\sim 8$ to $12 R_{E}$, where the current reduction occurs, while two outer probes, one at 20 and one at $30 R_{E}$, monitoring plasma acceleration due to magnetic reconfiguration such as tail flux re-connection and/or field dipolarization. Detailed design of the THEMIS mission, the satellites and their instrumentation is described by Angelopoulos (2008a) and references therein. The satellite observations are supplemented by a set of ground observatories in North America which measures the time of onset and the location of the aurora at substorm onset (Donovan et al., 2006; Mende et al., 2008).

The THEMIS satellites were launched on 17 February 2007 and they were all placed into their planned orbits to provide the desired tail sampling configuration by the winter of 2007-2008. During prior years the THEMIS ground based observatories GBO-s were built up and by the winter of 2007-2008 all 20 stations across northern America, 16 in Canada and 4 in Alaska, were operational. Thus the whole of the THEMIS concept of observations was fully implemented and data was collected during the conjunctions that occurred in the January to March of 2008 time period.

In this paper we will study the timing of substorm signatures using the ground and satellite based data from the THEMIS observations. We will discuss several events dur- ing two THEMIS conjunctions. We chose this set of conjunctions because of the good weather conditions over North America allowed us to monitor the detailed auroral displays over the relevant regions. We will first focus on the description of one of the events to demonstrate the various ground observables associated with substorm onset. Although such discussions are abundant in the literature (e.g. Akasofu, 1964; Lyons et al., 2002; Mende et al., 2007; Donovan et al., 2008) we focus our discussion towards using the ground based data to establish substorm onset timing with sufficient accuracy to be useful in making significant timing comparison required by the THEMIS goals. Following that we will use the satellite magnetic data to find substorm related magnetic impulses and compare their time of arrival to determine the time and location of their origin in the magnetosphere. We use the magnetic signatures because they have much longer-range effects than particle signatures and their propagation speed can be readily calculated from simple assumptions of particle density and magnetic field strength. In rare cases where the satellites were in the central plasma sheet particle measurements can be used to calculate the flow velocity and direction and the properties of the onset region thus are derived directly (e.g. Angelopoulos et al., 2008b).

\section{Substorm timing for 2 February 2008 07:00-09:00 UT}

On 2 February 2008 the THEMIS satellite constellation was in a favorable position to observe the geomagnetic tail region during several substorms as illustrated in Fig. 1. During the period between 07:00 and 09:00 $\mathrm{h}$ the THEMIS GBO-s showed that there were 3 major activation of the aurora at 07:40, 08:15 and 08:36 UT. The ground-based observations of these activations will be discussed in more detail later.

It is remarkable that the farthest satellite THEMIS B, located far in the magneto-tail at $29.6 R_{E}$ geocentric distance saw clear magnetic signatures associated with every one of these events. Apparently magnetic signatures of the events are longer range because there is no in situ plasma signatures associated with these events except with the last event occurring at 08:36 UT. Several minutes after this event there is evidence of strong earthward ( $+x$ blue) plasma flow. During the whole period presumably the relatively large GSM z coordinate of THEMIS-B the satellite shows that they are mostly located below the neutral sheet and only the relatively long range magnetic signatures are seen. Nevertheless these signatures can be very important in relating the timing of onset phenomena.

In the following discussion we will describe the three events and the associated ground based observations. For each event we will observe the magnetic signatures of the substorm onset at each satellite and will attempt to derive the timing and the $\mathrm{x}$ coordinate of the initial event. If two of the satellites were lined up along the neutral sheet and an event occurred between the two then it would be possible to 


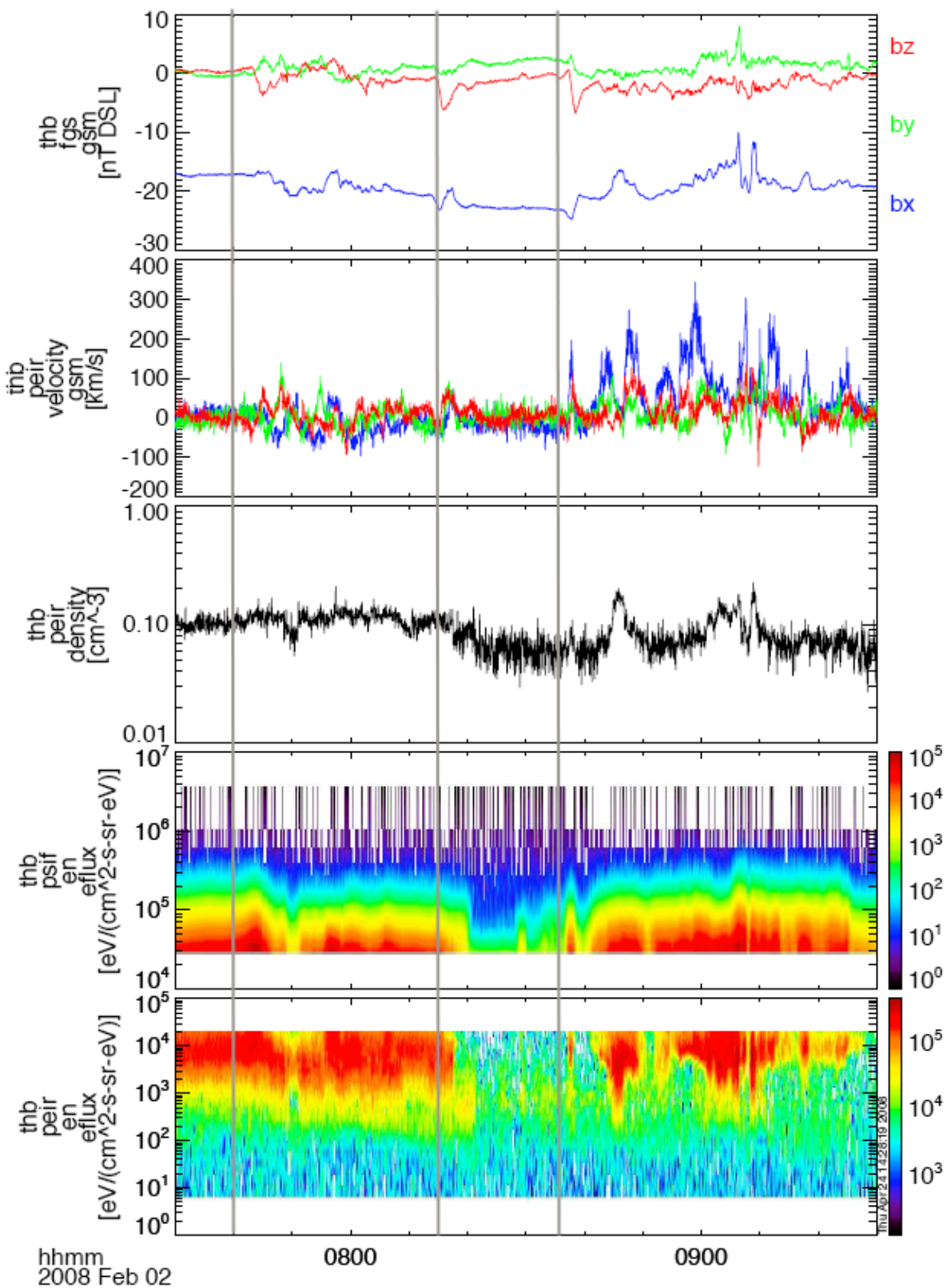

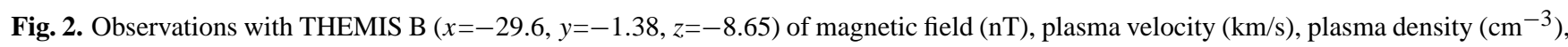
higher and lower energy ion spectrogram on 2 February 2008 as a function of UT.

calculate the time of and $\mathrm{x}$ location of the onset provided the velocity of the magnetic signal is known. If the velocity of propagation is a constant then we can simply calculate the start position $x_{0}$ and start time $t_{0}$. One of the waves traveling with velocity $v_{1}$ will reach the outer satellite located at distance $d_{1}$ at time $t_{1}$ and the other wave traveling with $v_{2}$ in the opposite direction will reach the inner satellite at distance $d_{2}$ at time $t_{2}$ then the starting time $t_{0}$ is related by the following equation

$v_{2}\left(t_{2}-t_{0}\right)-v_{1} *\left(t_{1}-t_{0}\right)=d_{2}-d_{1} \ldots$
In the case where the starting point is in between the satellites located at $d_{1}$ and $d_{2}$ respectively and the propagation velocity is constant $v=-v_{1}=v_{2}$ we get the result that the starting time is:

$t_{0}=\left(\left(d_{1}-d_{2}\right) / v+\left(t_{1}+t_{2}\right)\right) / 2 \ldots$.

If the starting point is not located between the two the satellites and $v=v_{1}=v_{2}$, i.e. the direction of propagation is the same, then Eq. (1) becomes:

$v=\left(d_{2}-d_{1}\right) /\left(t_{2}-t_{1}\right)$ 


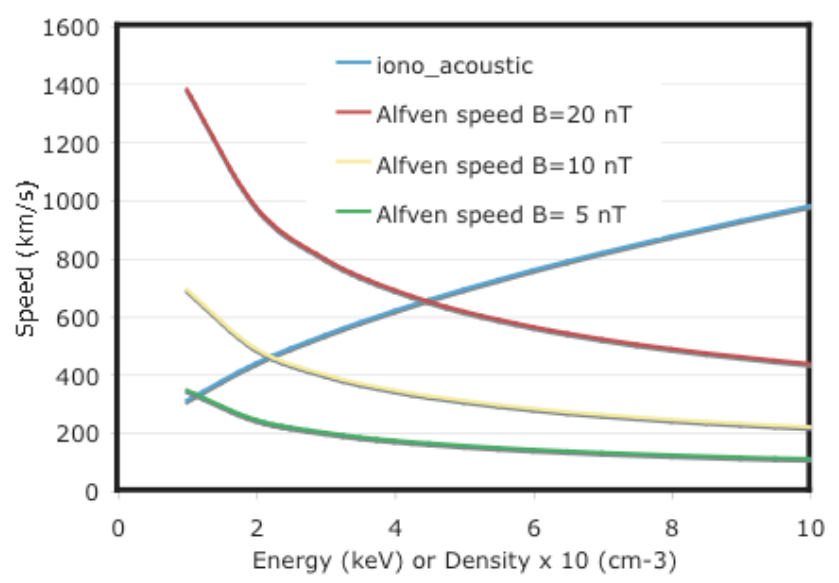

Fig. 3. Iono-acoustic and and Alfvén velocities as a function of plasma parameters. The energy scale refers to the iono-acoustic velocity while the plasma density to the Alfvén Wave curves. Plasma sheet densities at $>8 R_{E}$ are usually less than $1 \mathrm{~cm}^{-3}$ (labeled as $10)$.

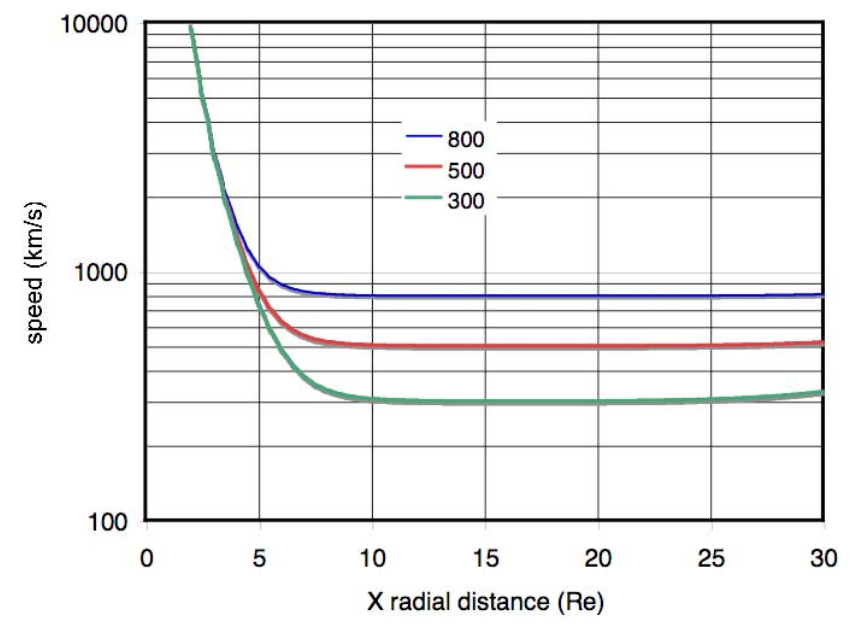

Fig. 4. The propagation speed as a function of the radial distance, $X$ from the Earth along the neutral sheet using Tsyganenko 1989 and a plasma density of $0.3 \mathrm{~cm}^{-3}$. At distances $>10 R_{E}$ the ionoacoustic speed becomes dominant and we assumed three constant values 300,500 or $800 \mathrm{~km} / \mathrm{s}$.

This equation allows the computation of the propagation velocity but provides no information about where the waves had started. If we are reasonably confident that the starting point of the waves was located between the two satellites but

$v\left(t_{2}-t_{1}\right)>\left(d_{2}-d_{1}\right)$

Then the inequality shows that there had to be a timing error because during the time difference of the wave arrival at the satellite the waves should have covered a larger distance than the distance between the two satellites.

The propagation velocity in the magnetosphere is not a constant and is given by $V_{F M}=\left(V_{A}^{2}+V_{s}^{2}\right)^{1 / 2}$, where $V_{S}$ is the

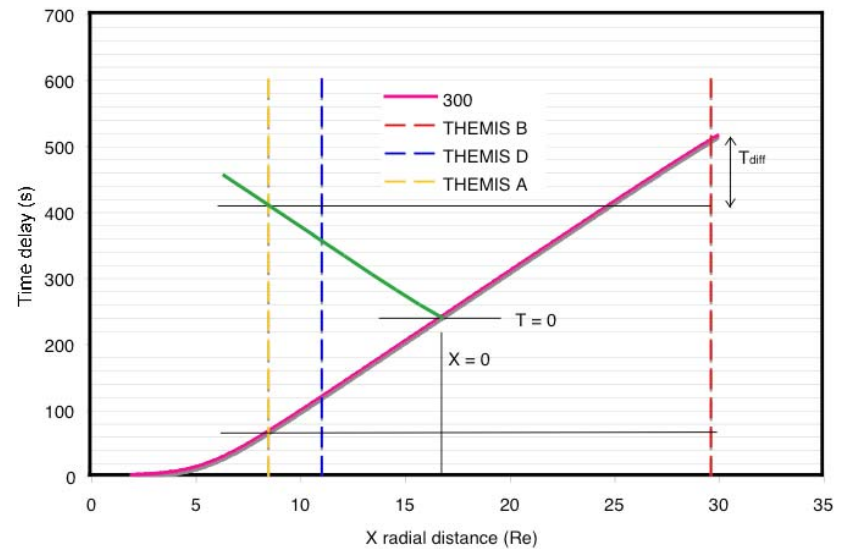

Fig. 5. Illustration of obtaining the $X=0 T=0$ graphically from the difference in arrival times ( $\left.T_{\text {diff }}\right)$, the x position of the satellites and the curve representing the integrated reciprocal of the propagation velocity (using the $300 \mathrm{k} / \mathrm{s}$ case) as a function of radial distance.

ion acoustic speed $=(\mathrm{kT} / \mathrm{m})^{1 / 2}$ and $V_{A}$ is the Alfvén speed $=\mathrm{B} /(4 \pi \mathrm{nm})^{1 / 2}$ (e.g. Moore et al., 1987). Both speeds can be calculated by making a few assumptions. From Fig. 2 satellite B seems to have briefly encountered the plasma sheet at about 08:45 UT and the measured density (panel 3) was about $0.2 \mathrm{~cm}^{-3}$ with a strong $B_{x}$ component of about $20 \mathrm{nT}$. Figure 3 show examples of the iono-acoustic speed as a function of the thermal energy associated with the plasma expressed in $\mathrm{keV}$ and the Alfvén speed as function of the plasma density (x10) with the ambient magnetic field being used as a parameter.

In calculating the Alfvén speed we used a combination of the IGRF internal reference field combined with the Tsyganenko 1989 external field in a low magnetic activity condition. Within the neutral sheet itself the $B_{x}$ component is very small and the models show that $x>10 R_{E}$ the $V_{F M}$ is dominated by the ion-acoustic speed. We have used a constant plasma density of $0.3 \mathrm{~cm}^{-3}$. This is consistent with THD and THE density measurements. kT seen by this satellite was approximately 2000 to $3000 \mathrm{eV}$, which would yield an ion acoustic speed estimate of about $500 \mathrm{~km} / \mathrm{s}$. The assumption of using the Alfvén/Magnetosonic speed itself can also be questioned on account of plasma inertia and the cumulative effects of reconnection forces. It is hard to know what the average propagation speed of these features is from the location of their generation to the probes where they were measured. Propagation speeds can be estimated from the measured ion speeds at THA, THD, at the time of dipolarization. From the flow velocity measurements of the THD energetic ion moments the flow velocity seemed to reach about $200 \mathrm{~km} / \mathrm{s} \mathrm{im}$ mediately after dipolariation. We recognize that the assumed propagation speed will have a profound effect on our result and we have therefore tried to bracket the problem by showing how the propagation speed affects the results. 
Figure 5 illustrates another possible graphical method for obtaining a starting location $(x=0)$ and time $(T=0)$ from measurements of the time difference of arrival of the magnetic impulse at two satellites with varying propagation velocity. The red curve is a derivative of the propagation speed curve of Fig. 4 corresponding to an iono-acoustic speed of $300 \mathrm{~km} / \mathrm{s}$. In this plot we have integrated the reciprocal of velocity along the radial distance to produce the time taken by signals propagating along this dimension. In this plot the ordinate represents a linear time dimension where simple linear equations can be solved for obtaining the starting location and time regardless of the intrinsically non-linear nature of the propagation velocity.

As an example consider that a substorm was observed by satellite THEMIS A (located at $8.5 R_{E}$ yellow vertical dashed line) and the signature of the same substorm was picked up at THEMIS B (located at $29.5 R_{E}$ red vertical dashed line) at $T_{\text {diff }}$ later. Assuming that the signature had started at $X=0$ and $T=0$ and "propagated along" the red curve left to right to THEMIS B. We can subtract $T_{\text {diff }}$ from the ordinate value at THEMIS $\mathrm{B}$ and draw a horizontal line to the $\mathrm{X}$ radial location of THEMIS A. The ordinate of this line represents the time when the signal was seen by THEMIS A. If we were to propagate back toward red curve at the speed the signal is traveling from $X=0$ to THEMIS A then we could find the point representing $X=0$ and $T=0$. To find the appropriate right to left propagation speed we need to reflect the red propagation curve about a horizontal axis and move it vertically up and down until it passes through the intersection of the previously drawn horizontal line and the $\mathrm{X}$ position of THEMIS A (vertical yellow dashed line). We show this as a green curve. The intersection of the reflected green curve with the original red curve provides the point of origin of substorm $X=0$ and $T=0$.

It is interesting to note that if $T_{\text {diff }}$ has to be less than the difference between the ordinates at THEMIS B and THEMIS A to obtain a solution for $X=0, T=0$ between the two satellites. This is the same condition that was discussed with respect to Eq. (3) in the earlier timing discussion related to constant velocities.

The speed is essentially constant at distances much larger than $10 R_{E}$. In addition to the graphical illustration presented here we have used an iterative non-linear treatment to allow for variable propagation speed (see Fig. 4) and used a non linear iterative computation for getting the starting location and times.

The first event under study is illustrated on Fig. 6 . The total integrated light curve gives a convenient quasiquantitative description of the substorm onset. We have identified four occurrences as distinct recognizable events, which characterize most substorm onsets as seen by the THEMIS all sky camera imagers. We have identified the first such event (1) as the pre-onset fading of the arc as seen by the dip in the intensity curve. Pre onset auroral fading has been identified many times in the literature, e.g. Heikkila and Peli-

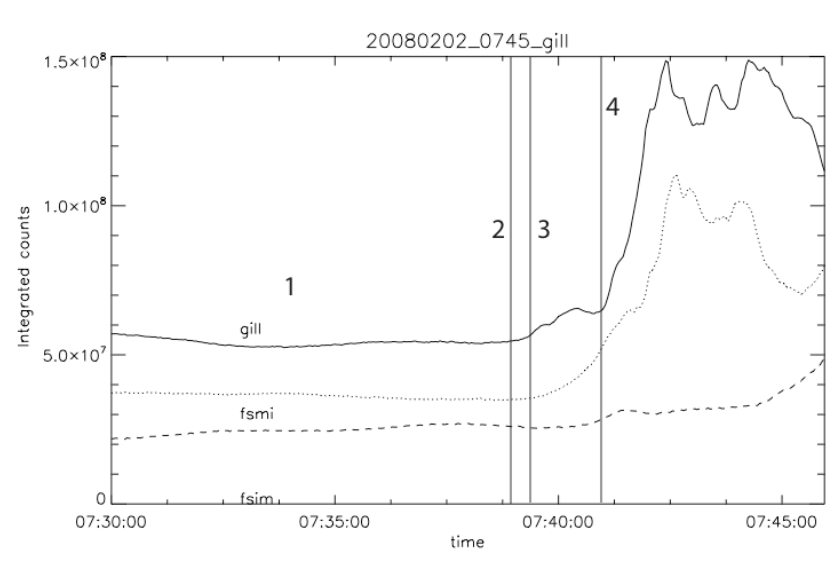

Fig. 6. Total light curves seen by the all sky imagers at Gillam Fort Smith and Fort Simpson. The various ground based onset events are shown with numerals 1 to 4 , where 1 is the pre-onset fading of the arc as seen by the dip in intensity, 2 is the time when the equatorward arc starts intensifying, 3 is the appearance of a new rayed arc feature and 4 is the onset of the Substorm Poleward Expansion (SPE) and rapid intensification of the aurora associated with the substorm.

nen (1972). The second pre-cursor event is the intensification of the pre-existing equatorward arc. The start of this period was noted with a vertical line (2). We believe that Akasofu in his original phenomenological definition of substorm onset, "the sudden brightening of an equatorward aurora near mid-night" (Akasofu, 1964) was indeed this brightening.

Unfortunately this intensification phase can last for several minutes and therefore it is difficult to use it as an exact time mark for the type of timing accuracy required to determine the step sequences critical to the THEMIS mission (Mende et al, 2007). The next step (vertical line 3 ) is the appearance of a new rayed arc. The appearance of a new arc at substorm onset has been described by others e.g. Lyons et al. (2002). This arc is highly structured with dynamic features looking much like Alfvénic aurora aurora as often seen at substorm onset (Mende et al., 2003; Lessard et al., 2006). The last event denoted with vertical line (4) is the onset of the SPE and rapid intensification of the aurora. This step is a relatively well defined and it does represent the initiation of the major energy dissipation in the substorm. This step also represents a magnetospheric configuration change, which is signified by the rapid poleward expansion of the bright aurora.

Figure 7 shows the various events described above as depicted by a mosaic generated from the all sky images only from stations where the weather was clear. It was assumed that all luminosity was emitted at $110 \mathrm{~km}$ altitude in the production of these mosaics. The all sky lens system produces substantial inherent distortions at the edges of the camera field of views and the production of such mosaics involves serious extrapolations and some method of dealing with overlapping regions from neighboring cameras (Mende 

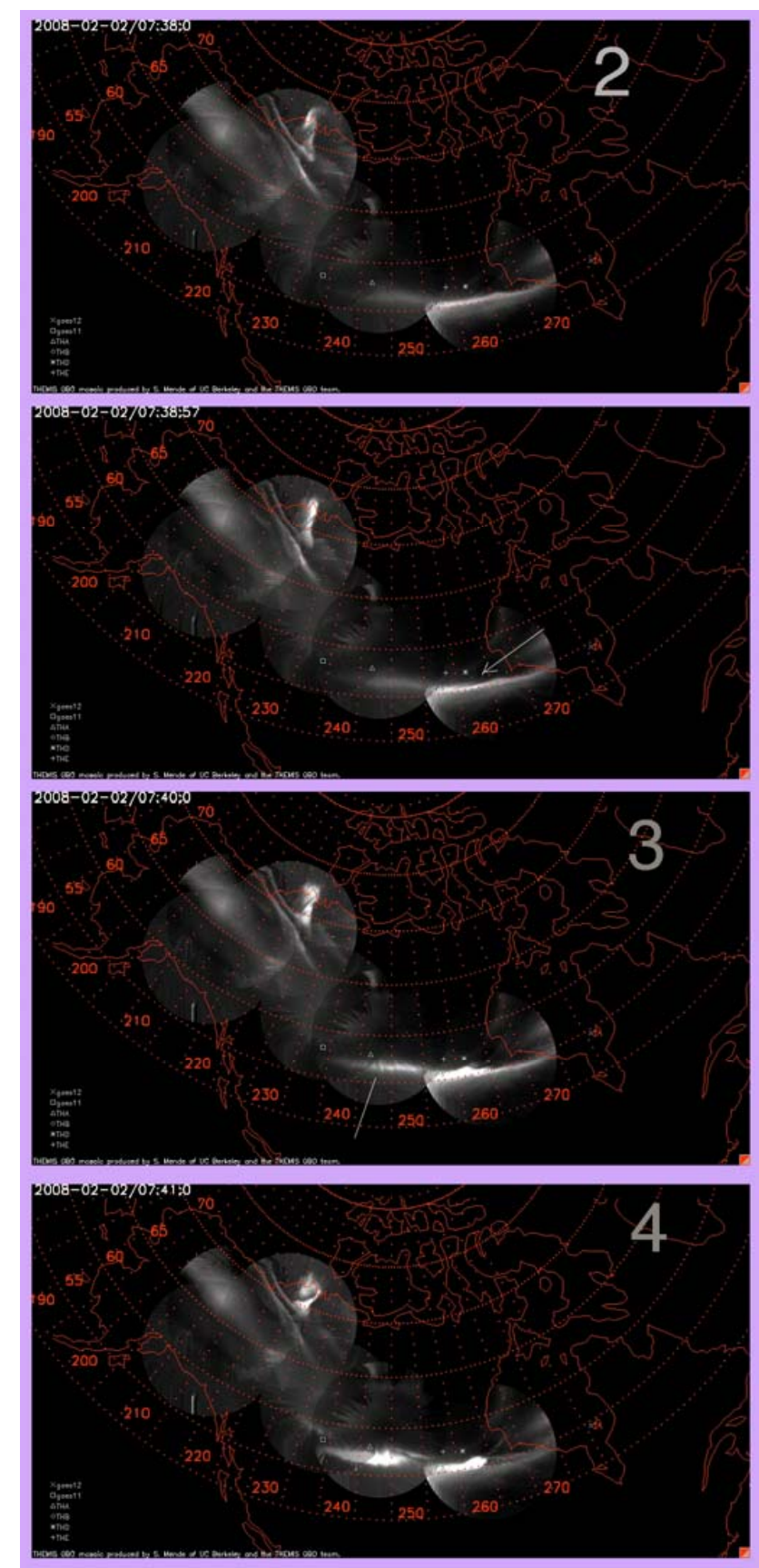

Fig. 7. Collage of image mosaics. Stations displayed from right to left are, Gillam, Ft. Smith, Ft. Simpson then Gakona (South), Ft. Yukon (middle) and Inuvik (North). The numerals on the top right are the identifiers of the various events as described in the caption of Fig. 4.

et al., 2008). A unique feature of this presentation (Fig. 7) is that overlapping regions were superposed by calculating a weighted average intensity of the intensities of the overlap. The weighting function was the reciprocal of the linear distance of the pixel from the central pixel of the station. This assured that any region was displayed with a resultant in- tensity which was dominated by the pixel intensity from the nearer station. This procedure created much greater continuity of the intensity of quasi-global auroral displays eliminating sharp boundaries between station field-of-views that are often discernable on mosaic displays.

At the time start of the sequence there was one auroral arc in the field of view of Gillam and Ft. Smith (Fig. 7 top panel) labeled "2". The arc faded at first (as seen on Fig. 6) and then intensified quite suddenly at 07:38:00 UT. The arc intensification was denoted as step 2 being the second step in the onset process. At 07:38:57 a new structure appeared located adjacent or just poleward of the pre existing arc. This is indicated with an arrow. In the subsequent frames (not shown) this region intensified and became quite prominent, The frame taken at 07:38:57 was chosen because that is the first frame where this feature could be distinguished. The next displayed frame (07:40:00) shows the rayed structure often associated with substorm onset aurora. The appearance of this aurora is very much like the Alfvénic auroras which have been seen at substorm onset (Mende et al., 2003; Lessard et al., 2006). The appearance of the Alfvénic aurora is step 3 marked the top right of the mosaic frame. The last step is the SPE, the substorm poleward expansion and large scale intensification and it is denoted as step 4 shown on frame 07:41:00.

Figures 8 and 9 are the corresponding ground based magnetometer data for the same time period as Fig. 6. Since the onset occurred closest to Gillam it has the largest response in its negative $B_{x}$ component representing a strong westward current. In terms of the four steps in the substorm onset process there is very good correspondence between step 2 and step 4 in the increase in the electrojet current strength and the auroral luminosity as seen in the all sky camera integrated light.

Figure 10 shows the THEMIS satellite magnetometer signatures for the $\sim 07: 40$ UT event. The onset of the non-linear intensification and poleward expansion in the optical aurora is at 07:41:00 as determined from the all sky imager data Fig. 7 labeled as (step) 4. It was shown with the vertical line spanning the plot. The magnetic signatures of the satellites occur in the sequence of THA, THD, THE and then THB. From the images it is possible to argue that azimuthally THA is closer to the "epicenter" of the intensification than D and E and the surge only envelopes the latitude of $\mathrm{D}$ and $\mathrm{E}$ a little later than that of A.

The choice of the magnetic signature of the "onset" at each satellite is open to interpretation. The magnetic signals for the inner satellites are relative impulsive and irregular containing high frequency components. They are likely to be caused by a mixture of the arrival of the magnetic signal and other responses due to the high speed Alfvén waves reflecting over relatively short distances near the Earth. Therefore generally we followed the same procedure we adopted for the ground based optical data and used the earliest point where significant $\mathrm{dB} / \mathrm{dt}$ was seen. For a distant satellite such as $\mathrm{B}$ at 


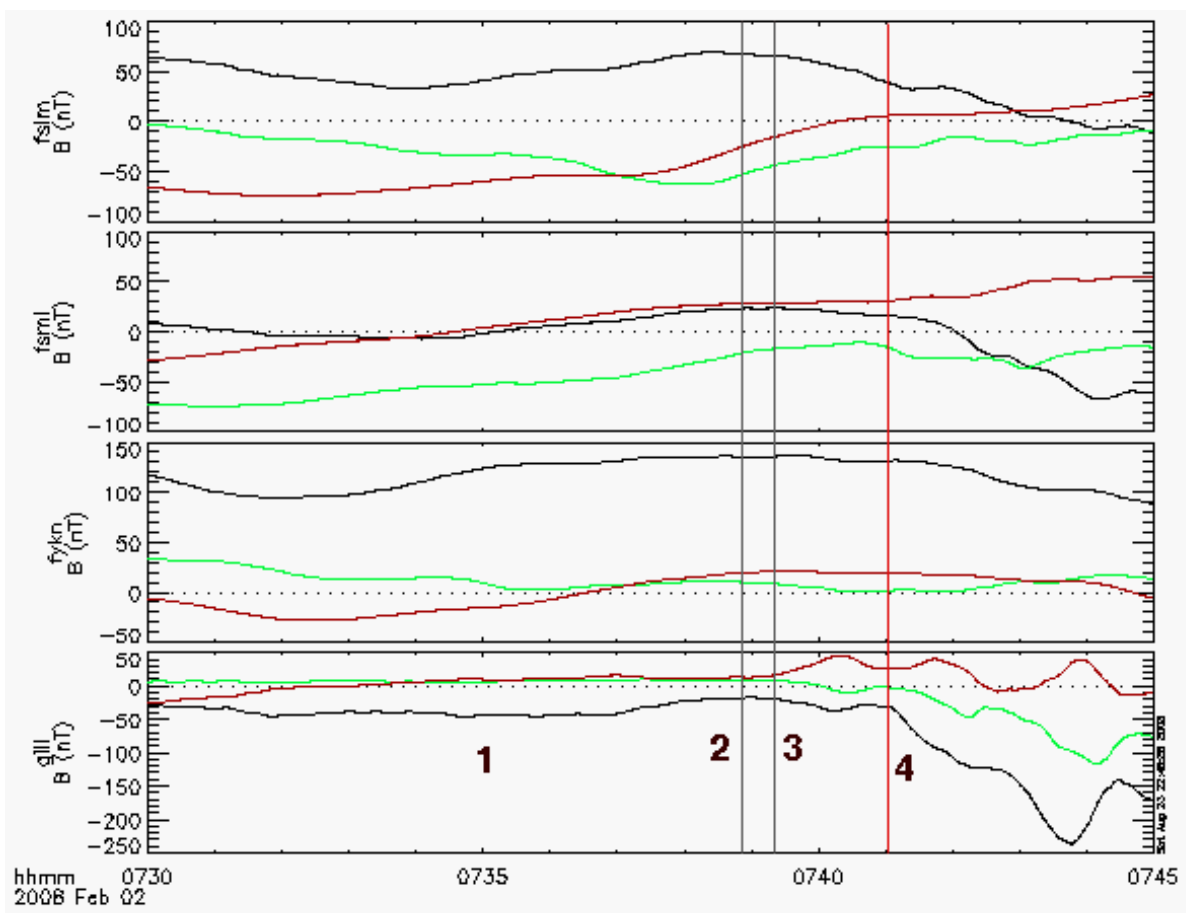

Fig. 8. The ground based magnetometer components, $B_{x}, B_{y}$ and $B_{z}$ (black, green and red) in full $0.5 \mathrm{~s}$ time resolution from Fort Simpson, Fort Smith, Fort Yukon and Gillam. The vertical bars represent the times identified in Fig. 6.

approximately $30 R_{E}$ down tail the magnetic signatures were relatively slowly varying signals consistent with a propagating magnetic structure slowly passing the satellite at some distance from the satellite. In such a case it is the large amplitude magnetic field change should be used for timing. Therefore when timing these satellites we generally ignored the slow rise of the field preceding the maximum slope and measured the timing when the magnetic signal reached a large value because that represents the time when the structure passes nearest to the satellite.

According to the above reasoning the magnetic signatures THA was taken at 07:40:00 shown with the short red line. For THD and THE, it seemed to occur just after 07:41:00 (the 07:41:00 line indicates the ground based onset of the poleward expansion). Admittedly these choices are somewhat arbitrary for example we ignored the small positive $B_{z}$ step seen with THD at 07:38:40. This could be taken to be a pre-cursor event similar to those existing in the optical aurora. As regards to determining the THB time of occurrence we used the large downward slope of the $B_{z}$ signature occurring at 07:43:39. Magnetic signatures are relatively long range and we would expect an extended response when they pass by in the vicinity of a satellite. We read the time when the magnetic signature became fairly well developed rather than at the earliest discernible impulse.

Assuming that the energy dissipation phase of the substorm starts at a single point in space and propagates in the $\mathrm{x}$ direction, both towards the earth and away from it, we calcu- lated the time and location of the onset using the arrival times of the magnetic signatures at two satellites. In these calculations we are using a simple 1-dimensional model that was discussed in prior sections of this paper. Comparing the arrival of the magnetic impulse at THEMIS B and THEMIS A we obtained consistent solutions for two iono-sonic speeds of 300 and $500 \mathrm{~km} / \mathrm{s}$ indicating that the start was at a point $x \sim-14.8 R_{E}$ and $11.1 R_{E}$ with a corresponding start time of 07:38:26 and 07:39:44 which is 154 and $76 \mathrm{~s}$ prior to the ground observed onset of the poleward expansion. For the $800 \mathrm{~km} / \mathrm{s}$ we did not get a consistent set of solutions. When we compared the arrival times at THEMIS B and E again using 300,500 and $800 \mathrm{~km} / \mathrm{s}$ then we got a consistent solutions for starting points at $x=-16.9 R_{E} x=-14.5 R_{E}$ and $x=-11.0 R_{E}$. The corresponding starting times were 07:39:10, 07:40:26 and 07:41:10. These onsets preceded the ground based onset of the SPE by 110,34 and in the last case the SPE onset preceded the onset by $10 \mathrm{~s}$. These results have been reproduced in Table 1.

To examine the inconsistency between the results obtained by comparing the propagation from THEMIS B to A and from THEMIS B to D can be best done by the graphical method originally presented in Fig. 5. Here in Fig. 11 we illustratred the curves describing integrated $1 / \mathrm{v}$ for 3 different ionoacoustic speeds. We can estimate the substorm start point, $X=0$ and $T=0$, by comparing the time of arrival of the signatures at two satellites and following the construction technique explained with reference to Fig. 5. By considering 


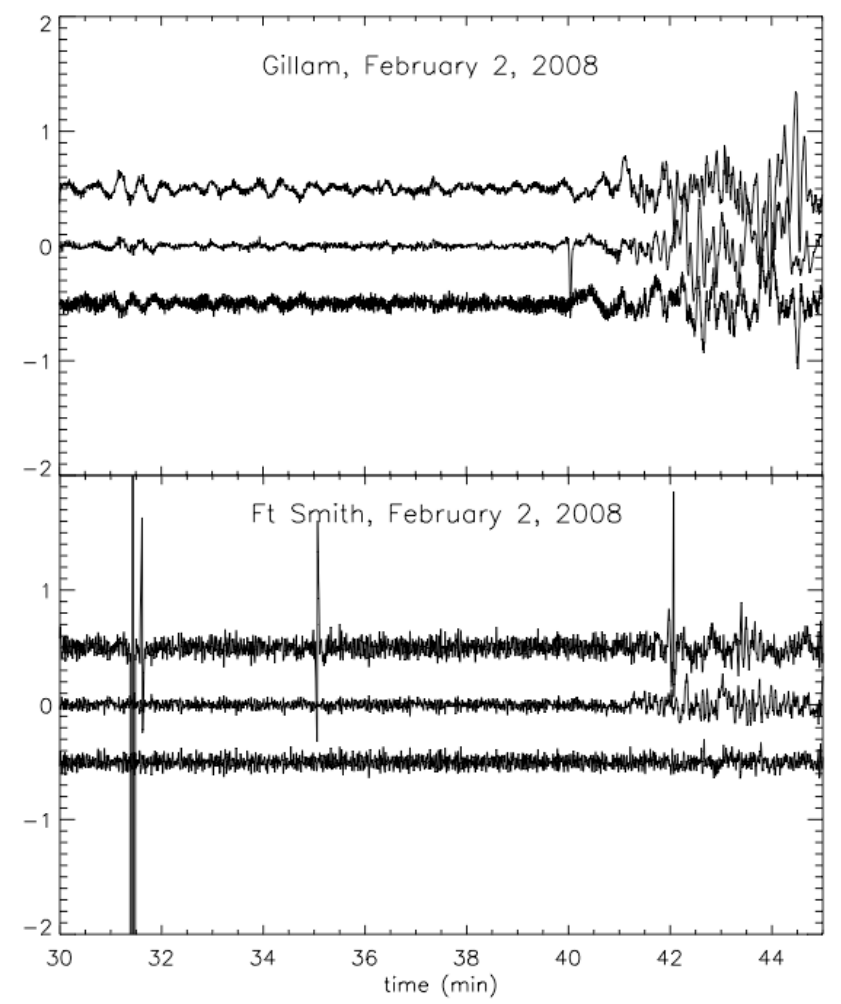

Fig. 9. Magnetic fluctuations $B_{x}, B_{y}$ and $B_{z}$ at Gillam and Ft. Smith. Larger amplitude fluctuations begin at Gillam at 07:40 UT.

first the time difference $T_{\text {diff }}$ between B and A (shown as a vertical black line on the right) and subtracting it from the ordinates at the position of THEMIS B we find that the $800 \mathrm{~km} / \mathrm{s}$ curve is too shallow and inconsistent with either the large a time delay or with having the position of the starting point between satellites B and A. Applying the technique to the $500 \mathrm{~km} / \mathrm{s}$ curve however, we obtain a starting point at about $11 R_{E}$-s as illustrated with the red circle. We were hoping to be able to get consistency using a third satellite. However when we considered $T_{\text {diff }}$ between THEMIS B and D and applied it to the $800 \mathrm{~km} / \mathrm{s}$ curve we obtained nice agreement with the starting point at about $11 R_{E}$ or so. However we had to rule out the $800 \mathrm{~km} / \mathrm{s}$ curve from the previous argument of being too large a velocity to account for the long delay between B and A. We applied the B to D time difference to the curve representing the velocity of $500 \mathrm{~km} / \mathrm{s}$. We got the intercept location of $14.5 R_{E}$ denoted by the blue circle. If we applied either time delays to the $300 \mathrm{~km} / \mathrm{s}$ curve then we would get intercepts at 14.8 and $16.9 R_{E}$ implying substorm starts further away from the Earth.

\section{Event occurring at 08:12}

The next event can be discussed in terms of Fig. 12. Ignoring an intensification that had occurred at 08:06 UT (seen at Gillam) the non-linear intensification and poleward expansion associated with this substorm occured around 08:12:48 UT at Ft. Simpson. Figure 13 shows the magnetometer data from the four stations and once again the $\mathrm{X}$ component traces show remarkable similarity to the total light curve. The first magnetic bay was seen at Ft. Simpson and the response at Ft. Smith was slightly delayed but actually reaching a higher intensity value than the Ft. Simpson negative bay. This delay and higher intensity behavior is quite similar to what was observed in the total light curve shown in Fig. 12.

Figure 13 shows that the negative bay at Ft. Simpson began simultaneously with the intensification of the total light curve. Both figures show that although the signature started at Ft. Simpson the resultant intensification of the strength of the magnetic bay was larger at Ft. Smith. The fact that the Ft. Smith signatures were delayed confirms that the event was propagating eastward.

In Fig. 14 we presented the magnetometer data for the four THEMIS satellites and in the caption we identified the time of arrival of the propagating magnetic signature. On Fig. 15 we present the auroral image collages representing the auroral display at the time of these signatures. Figure 15a depicts the auroral situation at the time of the expansion onset. Figure $15 \mathrm{~b}$ shows the aurora when the magnetic signature reaches THEMIS A. THEMIS A is in the correct local time region to see the onset. However THEMIS D and $\mathrm{E}$ are off to the east and therefore it is not surprising that they observe the magnetic signature a lot later. Figure 15 (c) shows the aurora when the magnetic signature reaches THEMIS B located at $29.5 R_{E}$ down tail. Figure $15 \mathrm{~d}$ shows the aurora when the magnetic signature reaches THEMIS D and E. By making the assumption that the substorm started between THEMIS A and THEMIS B we can calculate the onset $x$ location and the time of onset (see Table 1).

Kiehas et al. (2009) analyzed this same event and found that the magnetic signature at THEMIS B was consistent with their model of a "reconnection associated outflow region" traveling in the down tail direction. This is qualitatively consistent with our interpretation of the outward traveling magnetic signature at THEMIS B and their model predicts the onset location which is in agreement with the 17.6 $R_{E}$ shown in Table 1 for an assumed iono-acoustic speed of $300 \mathrm{~km}$. For the other speeds the predicted distance from the Earth is less.

For the second event the comparison between the arrival of the magnetic impulse at THEMIS B and THEMIS A yields self consistent starting points at $x=-17.6 R_{E}$, $x=-15.9 R_{E}$ and $x=-13.6 R_{E}$ wth corresponding starting times of $08: 10: 36,08: 11: 55$ and $08: 12: 42$ for assumed speed 


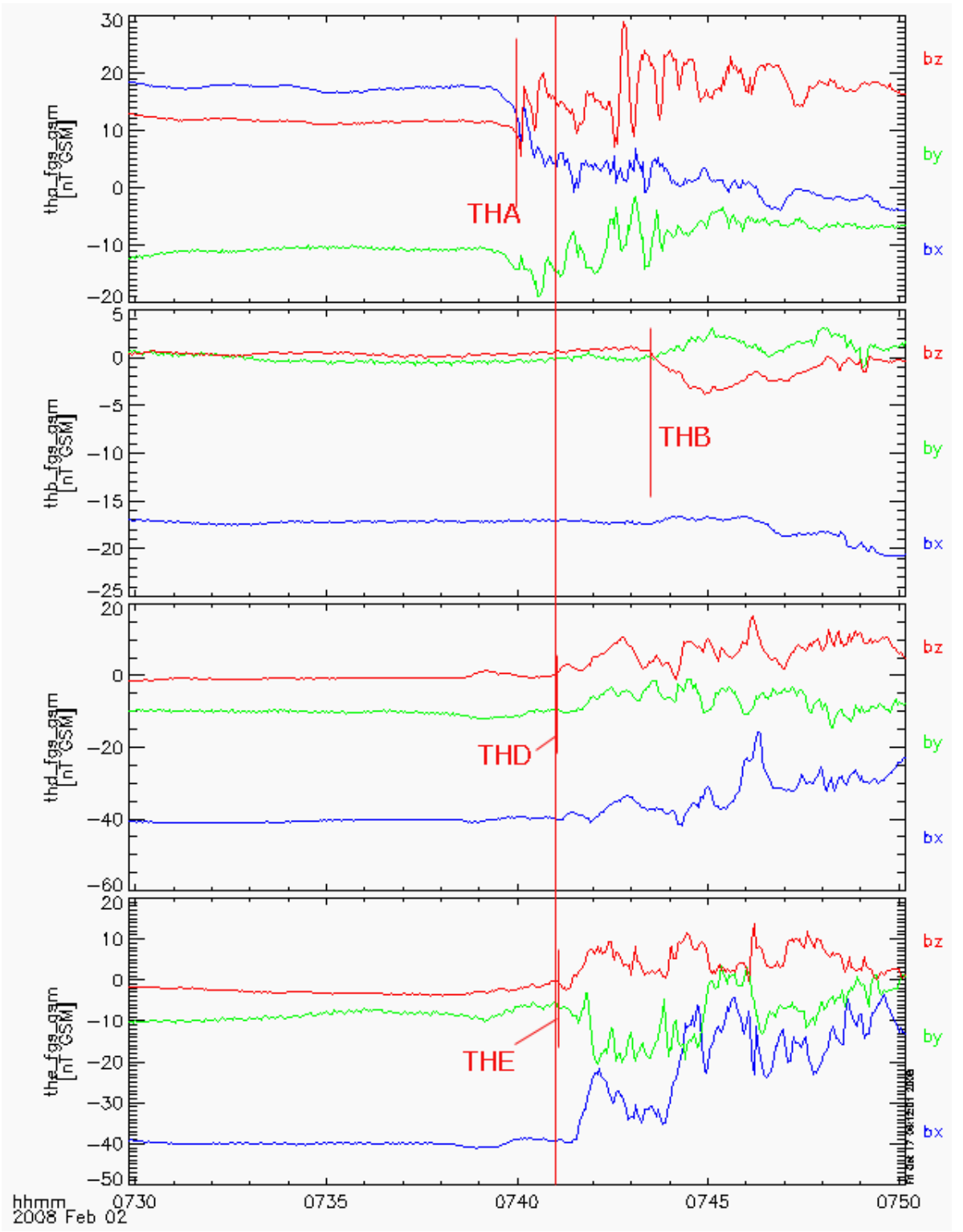

Fig. 10. THEMIS magnetometer data from THEMIS satellites A, B, D and E. The onset of the large scale intensification of the aurora as seen from the ground at 07:41 UT. The sequence of observations is THA, ground, THD, THE and THB.

of 300,500 and $800 \mathrm{~km}$, respectively. These results are also given in Table 1.

\section{Description of the $\sim 08: 35$ event}

The next event can be discussed in terms of Fig. 16. The non-linear intensification and poleward expansion (SPE) associated with this substorm occurred around 08:35:42 UT at Ft. Simpson. Figure 17 shows the magnetometer data from the four stations and once again the $\mathrm{X}$ component traces at Ft. Simpson show remarkable similarity to the timing of total light curve.
In Fig. 18 we presented the magnetometer data for the four THEMIS satellites and noted the ground based SPE signature with a vertical line at 08:35:42 UT. Accurate timing of the arrival of the magnetic signal at the satellite is clearly problematic and timing errors of the order of 10-20 s $\sim 1$ or $2 R_{E}$ are quite possible. We assumed that THEMIS B sitting out at $30 R_{E}$ distance and most likely located outside of the event would see a propagating magnetic structure going by. The slowly varying magnetic field signal is consistent with this and the timing therefore should be taken when the magnetic signal value is largest because that is when the structure passes nearest to the satellite. Thus we ignored the slow rise of the $B_{z}$ field between 08:36:00 and 08:37:20. We 
Table 1. Timing results from 3 substorms on 2 February 2008. First column shows substorm number designation and ground based determination of time of Substorm Poleward Expansion (SPE) onset or step 4. Second and third columns THEMIS satellite designator and time of arrival of magnetic impulse. Fourth column is the assumed ionoacoustic speed for the calculations. Fifth and sixth columns are the computed time (time delay to SPE in minutes and seconds) and x distance of the "point of origin" of magnetic impulse from earth respectively.

\begin{tabular}{|c|c|c|c|c|c|}
\hline $\begin{array}{l}\text { Substorm \#/Poleward } \\
\text { (SPE) expansion start }\end{array}$ & $\begin{array}{l}\text { 1st Satellite/time } \\
\text { of signature }\end{array}$ & $\begin{array}{c}\text { 2nd Satellite/time } \\
\text { of signature }\end{array}$ & & $\begin{array}{l}\text { Predicted onset time (time diff } \\
\text { from SPE) min:s }\end{array}$ & $\begin{array}{l}\text { Predicted onset distance } \\
\mathrm{X}\left(R_{E}\right)\end{array}$ \\
\hline \multirow{4}{*}{ 1/07:41:00 } & \multirow{4}{*}{ ТHB/07:43:39 } & \multirow{4}{*}{ THA/07:40:09 } & 300 & $07: 38: 26(2: 34)$ & 14.8 \\
\hline & & & 500 & $07: 39: 44(1: 16)$ & 11.1 \\
\hline & & & 800 & $07: 40: 08-53(<0: 52)$ & $<8.5$ \\
\hline & & & 300 & $07: 39: 10(1: 50)$ & 16.9 \\
\hline \multirow[t]{3}{*}{ 1/07:41:00 } & \multirow[t]{3}{*}{ THB/07:43:39 } & \multirow[t]{3}{*}{ THE/07:41:09 } & 500 & $07: 40: 26(0: 34)$ & 14.5 \\
\hline & & & 800 & $07: 41: 10(-10)$ & 11.00 \\
\hline & & & 300 & $08: 10: 36(2: 12)$ & 17.6 \\
\hline \multirow[t]{3}{*}{ 2/08:12:48 } & \multirow[t]{3}{*}{ THB/08:14:50 } & \multirow[t]{3}{*}{ THA/08:13:20 } & 500 & $08: 11: 55(0: 53)$ & 15.9 \\
\hline & & & 800 & $08: 12: 42(0: 6)$ & 13.6 \\
\hline & & & 300 & $08: 32: 53(2: 49)$ & 17.0 \\
\hline \multirow[t]{2}{*}{ 3/08:35:42 } & \multirow[t]{2}{*}{ THB/08:37:20 } & \multirow[t]{2}{*}{ THA/08:35:24 } & 500 & $08: 34: 12(1: 30)$ & 14.9 \\
\hline & & & 800 & $08: 34: 59(0: 43)$ & 12.0 \\
\hline
\end{tabular}

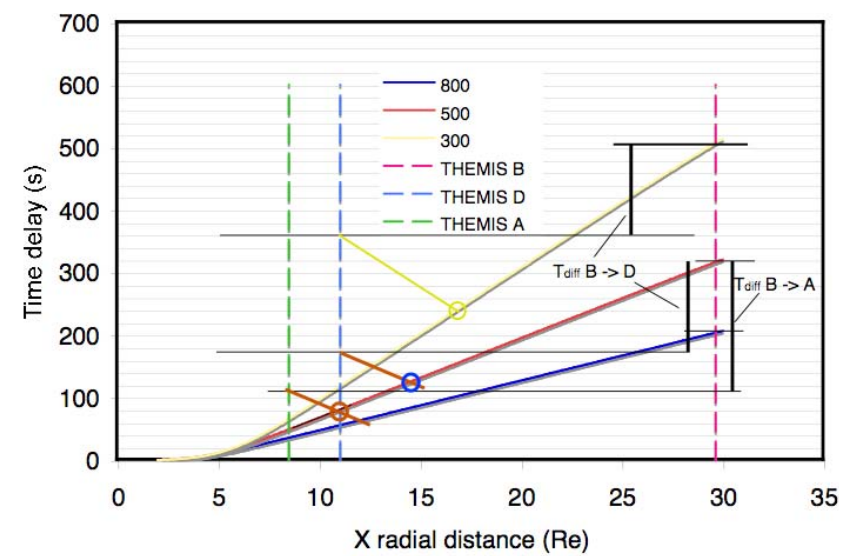

Fig. 11. The three curves represent models of the integrated $1 / \mathrm{v}$ with 300,500 and $800 \mathrm{~km} / \mathrm{s}$ iono-acoustic speeds (yellow, red and blue). The satellite positions for the 07:41:00 event of THEMIS A, D, and $\mathrm{B}$ are illustrated by the green, blue and red vertical dashed lines. The time difference $T_{\text {diff }}$ between the magnetic signature arrivals shown with the black vertical bars. Following the graphical method of deriving $X=0$ and $T=0$ from the signature arrival difference between satellite $B$ and A we can show that using the $500 \mathrm{~km}$ speed curve $X=0$ was at $11 R_{E}$ and is illustrated with a red circle. The blue and yellow circles represent the same calculation for the time difference between D and A for 500 and $300 \mathrm{~km} / \mathrm{s}$ curves, respectively.

used 08:37:20 for our timing when $B_{z}$ turned sharply negative. One may argue that for the maximum field signature one should have taken it a few more seconds later when $B_{y}$ was maximum and when $\mathrm{Bz}$ was halfway between its maximum and minimum. For the inner satellites we assumed that the satellite actually encountered the event. As the magne-

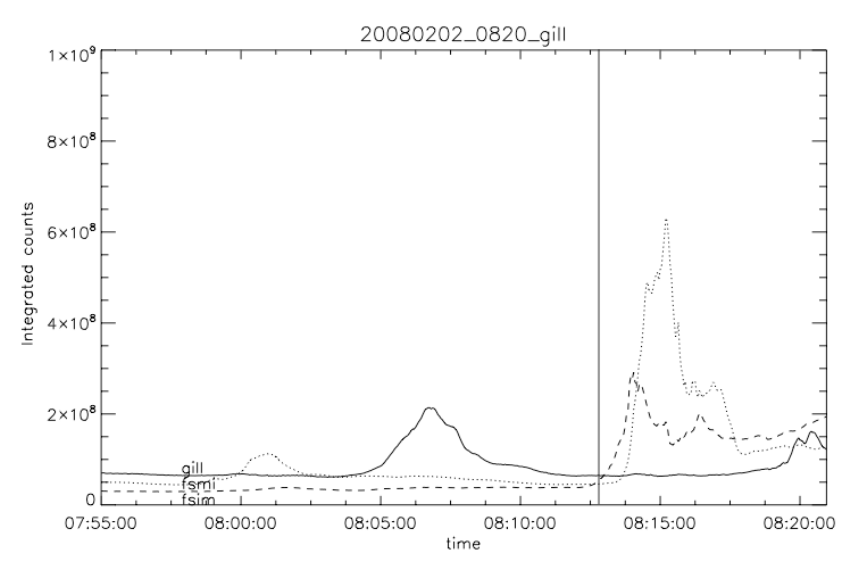

Fig. 12. Auroral luminosity curves for $\sim 08: 12$ event.

topshere is considerably "smaller" here at $\sim 10 R_{E}$ and the magnetic signals are a mixture of several nearby current system we had no choice but to use the "sudden impulsive" start of magnetic signature and we chose the sudden increase of the By component at 08:35:24.

On Fig. 19 we present the auroral image collages representing the auroral display at the time of these signatures. Figure 19a depicts the auroral situation at the time of the ground based expansion onset. Figure 19b shows the aurora when the magnetic signature reaches THEMIS A. THEMIS A is reasonably well placed to be near the local time region nearest the onset. Figure 19c shows the aurora when the magnetic signature reaches the foot point of THEMIS B located at $29.5 R_{E}$ down tail. Figure $19 \mathrm{~d}$ is the image collage of the aurora when the magnetic signature reaches THEMIS E and D. 


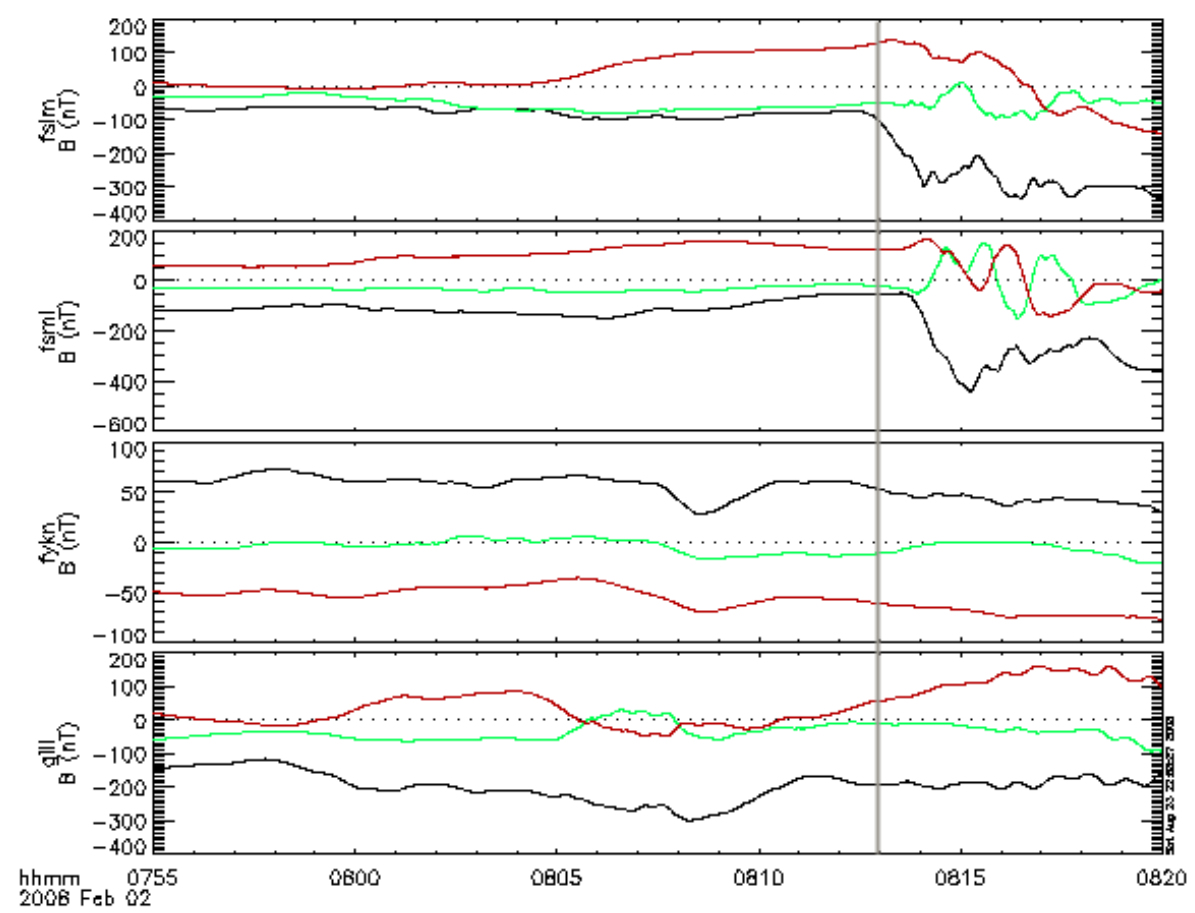

Fig. 13. Ground magnetometer data $B_{x}, B_{y}$ and $B_{z}$ (black, green and red) associated with the second $\sim 08: 12$ event.

By making the same assumption that the substorm started between THEMIS A and THEMIS B we calculated the onset $\mathrm{x}$ location and the time of the onset for the third event. The time comparison between the arrival of the magnetic impulse at THEMIS B and THEMIS A yielded a consistent starting points at $x=-17.0 R_{E}$, at $x=-14.9 R_{E}$ and at $x=-12.0 R_{E}$ with corresponding starting times of 8:32:53, $8: 34: 12$ and 8:34:59 for the three assume speeds of 300,500 and $800 \mathrm{~km} / \mathrm{s}$, respectively.

\section{Discussion of the 29 January 2008 event}

As discussed previously we had to take strict account of the azimuthal (local time) positions of the THEMIS spacecrafts with respect to substorm onset. Otherwise the timing results could be flawed due to the delays caused by the aurora propagating azimuthally into the region of the THEMIS satellites. We note here that Lui et al. (2008) discussed the timing of several events that occurred on 29 January 2008 with relatively little regards to their relative local time position to the THEMIS satellites. They based their analysis on preliminary optical data and they identified three onset events at $\sim 07: 14$, $\sim 07: 42,08: 11$ from the individual images in the 07:00 UT to 09:00 UT period. They used these ground based onsets and they attempted to do a preliminary qualitative timing analysis of the magnetic and plasma flow data. We have attempted to re-analyze the same events using the techniques described above. However examination of the global mosaic data of these events showed that these events were not suitable for this type of timing analysis.

The first event ( $\sim 07: 14)$ had a significant auroral brightening but the auroral arc did not show the classic substorm poleward expansion phase. In view of this it is debatable whether this auroral intensification was really a substorm. The second event did show the classic poleward expansion and our global mosaic of the aurora associated with the onset intensification is illustrated in Fig. 20 taken at 07:43:00 two frames after the first sign of the poleward expansion at 07:42:42. This figure shows the onset location was at Ft Smith and only THEMIS A and GOES 11 satellites had their foot points near the onset local time or longitude. At 07:44 tailward moving event is seen at THB however the magnetic impulse signature at THA is seen only at 07:46. The substorm aurora reached $\mathrm{C}$ and D only at 07:53:27. These delays are more or less consistent with the local time propagation of the auroras from the onset point to the location of the magnetic foot point of the satellites and are too late to provide any sensible timing measurement related to propagation in a direction parallel to the $\mathrm{x}$ axis. A third major event started in Alaska and the THEMIS satellites were located too far eastward to be of any use in timing the onset initiation. Because of this difficulty we were not able to derive any onset timing based on the time of arrival of signatures at the satellites. In summary the 29 January set of events (Lui et al., 2008) are examples which were not suited for quantitative timing analysis and their results ignore the errors caused by the local time longitude propagation of the substorm. 


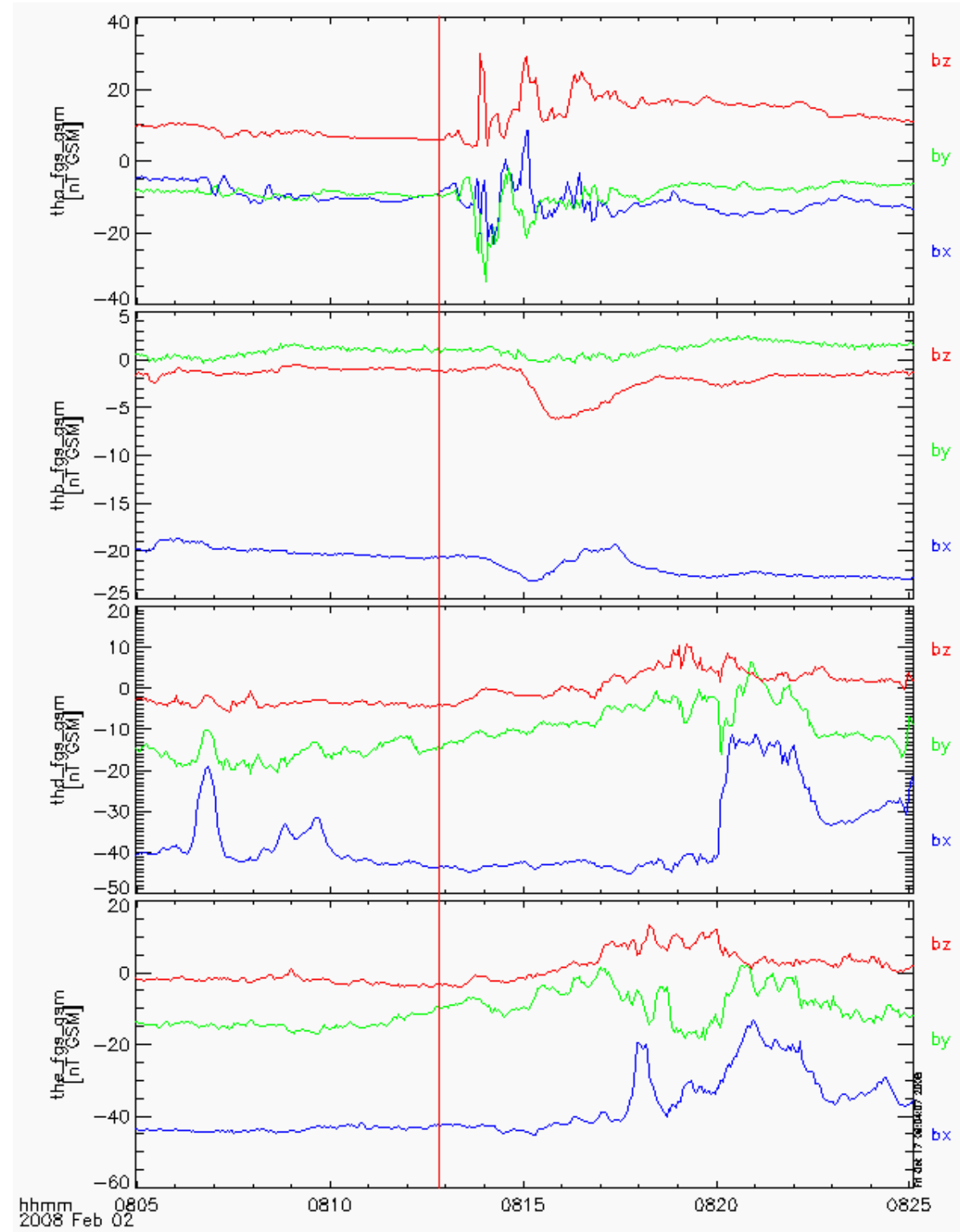

Fig. 14. Satellite flux gate magnetometer data. The red vertical denotes the nominal onset of the poleward expansion (SPE) at 08:12:48. The onset of the magnetic variation attributable to this substorm started at 08:13:21 for THEMISA, 08:14:50 for THEMIS B and at $~ 08: 17: 00$ for THEMIS D and E.

\section{Discussion}

We discussed 3 events that occurred on 2 February 2008 in detail where the azimuthal (local time) positions of the THEMIS satellites and of the substorm onset were closely aligned. As a counter example we briefly discussed another set of events which occurred on 29 January 2008. Both sets of events occurred during major conjunctions of the THEMIS satellite constellation and when reasonably clear weather permitting the observations of the Northern Hemisphere auroral zone with the THEMIS GBO chain. These were also moonless nights.
The first event on 2 February occurred at about 07:40 and we described the onset development in detail to illustrate the steps that constitute substorm onset as observed by the THEMIS GBO network. We found that for accurate timing the best marker was the start of the expansive phase substorm poleward expansion (SPE) because it is readily observable through the sudden non-linear growth in total light intensity accompanied by the poleward expansion. Nevertheless we also found that there are significant pre-cursor or trigger events that are unmistakably substorm associated features. The first one of these is the pre-onset fading of the auroral arc (Pellinen and Heikkila, 1978). A relatively 

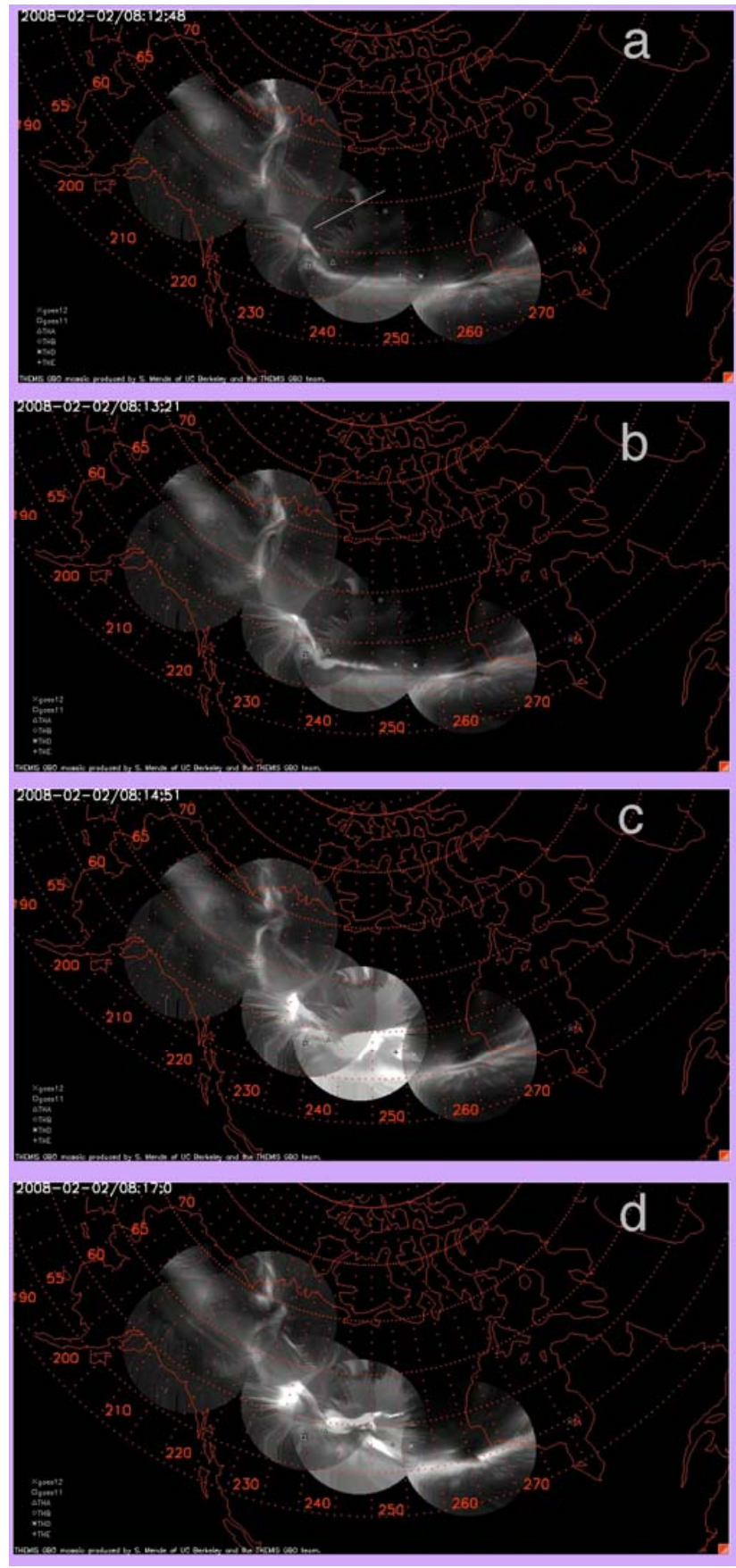

Fig. 15. (a) Onset at 08:12:48; (b), (c), and (d) the auroral image collages taken at the time when the magnetic signature is seen at the location of the satellite THEMIS A $(08: 13: 21)$ THEMIS B and (08:14:50) and THEMIS D and E (08:17:00).

brief time interval later the pre-existing arc intensifics which was termed as the onset of the expansive phase by Akasofu (1964). However this arc intensification is gradual and it can take several minutes and it is somewhat difficult to use it as a timing signature. Following that step we distinguish the appearance of a highly dynamic new feature (Lyons et al.,

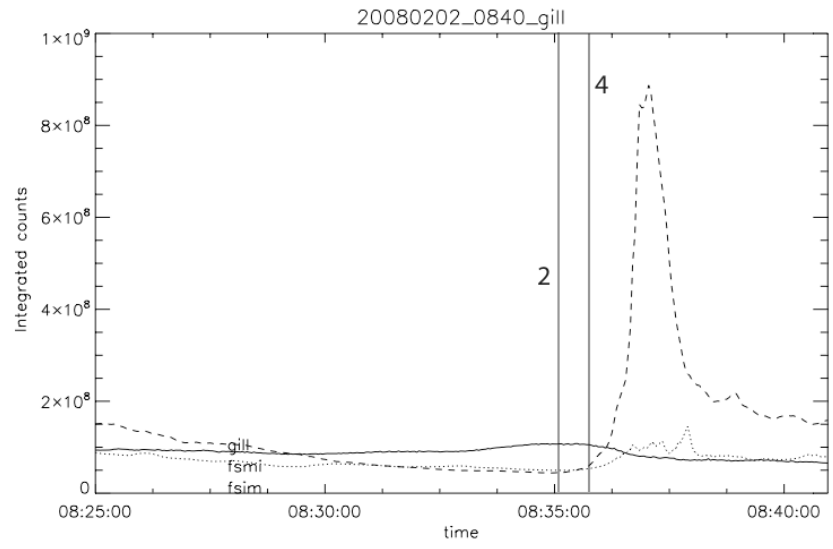

Fig. 16. $\sim 08: 35$ UT event. Strongest signature is at Ft. Simpson. Vertical line labeled 2 represents the arc intensification and 4 shows the onset of the large scale nonlinear intensification and accompanying substorm poleward expansion (SPE).

2002) that suddenly appears usually on the poleward side of the pre-existing arc. The dynamic nature and rayed appearance of this arc suggests that it may be an Alfvénic aurora of the type observed at substorm onset by Mende et al. (2003) and Lessard et al. (2006). We call these features pre-cursor phenomena because they precede the SPE which is the sudden large scale intensification and poleward motion of the substorm aurora. Our findings also show that some of these pre-cursor events precede the substorm onset in the magnetosphere as indicated by the satellite data. However these precursor events do not occur randomly and are definitely substorm associated. These events therefore provide evidence that in the substorm triggering phase the magnetosphere is closely tied to the polar ionosphere. The substorm instability, its energy storage and release is likely to be the result of a yet poorly understood trigger action of the closely coupled magnetosphere ionosphere system.

In addition to the practical advantage for using the SPE for accurate timing, the sudden non-linear growth in total light intensity represented by the SPE and the accompanying rapid poleward expansion signifies the start of the substorm energy dissipation phase in the ionosphere by precipitation and electrojet heating. The SPE signifies the period where the large scale energy release begins requiring simultaneous large scale magnetic re-configuration to supply the needed magnetic energy for particle acceleration.

We have derived the location and time of the substorm onset in the magnetosphere using satellite data independent of ground based observations. By using Tsyganenko 1989 magnetic field and plasma density distribution model corroborated by the THEMIS satellite plasma measurements we calculated the propagation speed, $V_{F M}=\left(V_{A}^{2}+V_{S}^{2}\right)^{1 / 2}$ of magnetic impulses in the magnetosphere. This speed results in a speed very close to the magnetosonic speed at distances greater than $10 R_{E}$. The Alfvén/Magnetosonic speed should 


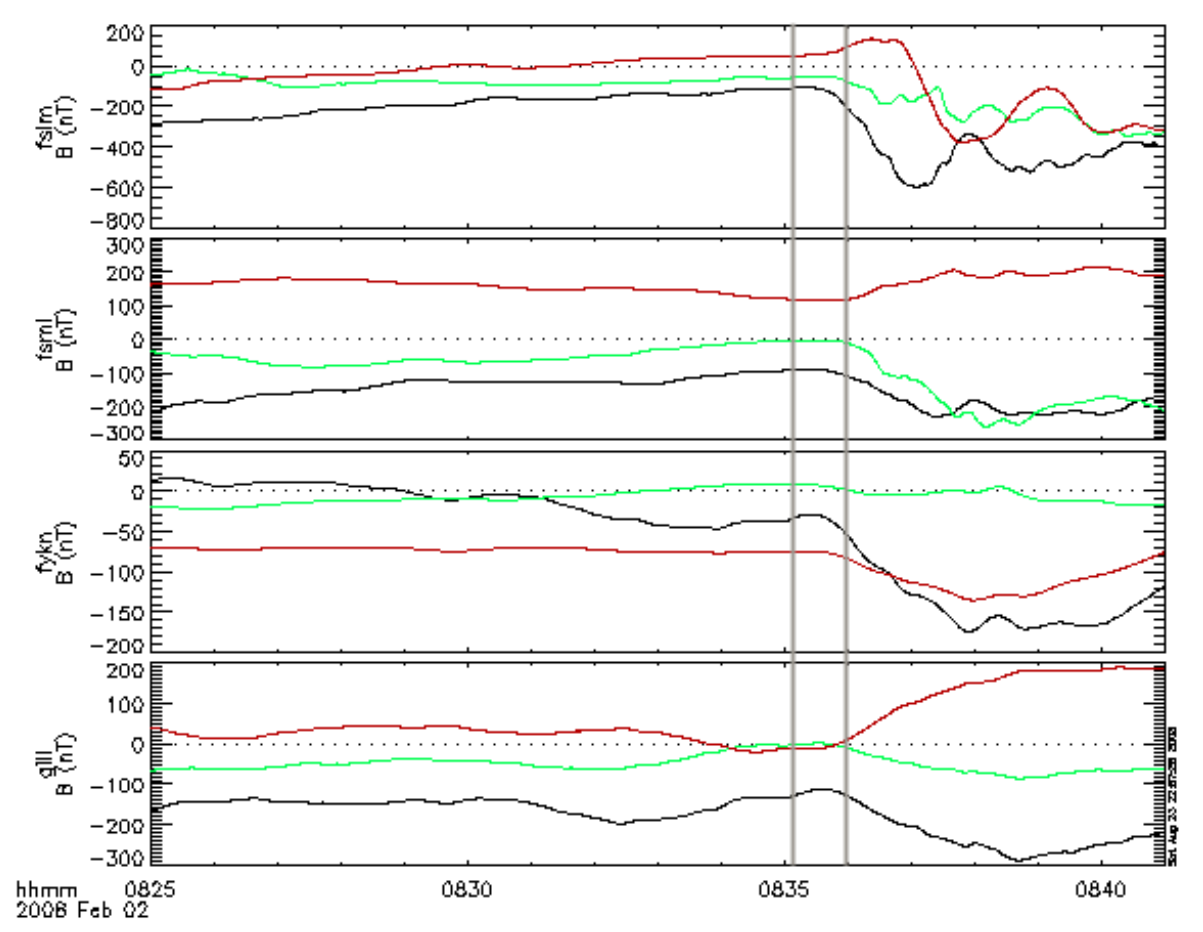

Fig. 17. Ground base magnetometer data shows the largest magnetic signature at Fort Simpson in agreement with the optical data.

be limited by plasma inertia and the cumulative effects of reconnection forces, furthermore the THEMIS D energetic ion moments flow velocity measurements at the dipolarization showed only a max velocity of $200 \mathrm{~km} / \mathrm{s}$. In the absence of reliable data we adopted three values for the magnetosonic speed 300,500 and $800 \mathrm{~km} / \mathrm{s}$ and used a simple onedimensional (parallel to the $\mathrm{x}$-axis) model of propagation of the magnetic impulses to two satellites from an unknown onset region located between the two satellites. In our software model we used an iterative approach of trying different the onset locations between two of the spacecrafts until the arrival times in the models fitted the observed arrival times. This approach allowed for a non-linear, variable speed propagation of the magnetic impulses. The results are tabulated in Table 1.

Accurate timing of the magnetic signal at the satellites is somewhat subjective and timing errors of the order of 10$20 \mathrm{~s}$ or about $1-2 R_{E}$ are quite possible. The initial magnetic signatures of the far away satellites such as THEMIS B were relatively slowly varying signals consistent with a propagating magnetic structure passing by the satellite at some distance from it. For the inner satellites the magnetic signatures were considerably sharper representing sudden magnetic activity at the near vicinity of the satellite. Considering this we justified using the observation of a large amplitude with significant $\mathrm{dB} / \mathrm{dt}$ for timing the arrival of the signal to the vicinity of the satellite and ignored slowly varying precursor signals.
In addition to the uncertainty of the magnitude of the propagation speed it is important to remember that even a single point of origin is questionable because if the timing at the outer satellite (THB) represents the plasmoid core going by and the magnetic impulse at THA represents the passing edge of the dipolarization, it is unlikely that they would have been launched from the same location. The core of the plasmoid would have been accelerated probably a few Re tailward of the $\mathrm{X}$ point and the dipolarization (Earthward of $\mathrm{X}$ the point) are closer than the $\mathrm{X}$ line. However compared to the relatively large separation between THA and THB these are small, $1-2 R_{E}$, errors.

In some cases especially for THEMIS D and E the longitudinal offset between the observed auroral onset region and the magnetic foot print of a THEMIS satellite was too large for our analysis because the aurora took a considerable time to reach the local time (longitude) region of the satellite foot print. In such cases we tried to use a linear correction scheme to take account of the azimuthal (longitudinal) propagation of the substorm aurora by deriving the added time delay from the time taken by the visible aurora to reach the satellite foot print. However we were not successful in getting consistent results in such cases.

In the cases discussed where the longitude of the satellite foot points were reasonably close to the auroral onset region as seen from the ground consistent results were obtained and the calculated location of the onset regions were surprisingly consistent for the 3 events and their position depended mostly on the propagation speed. These results need to be 


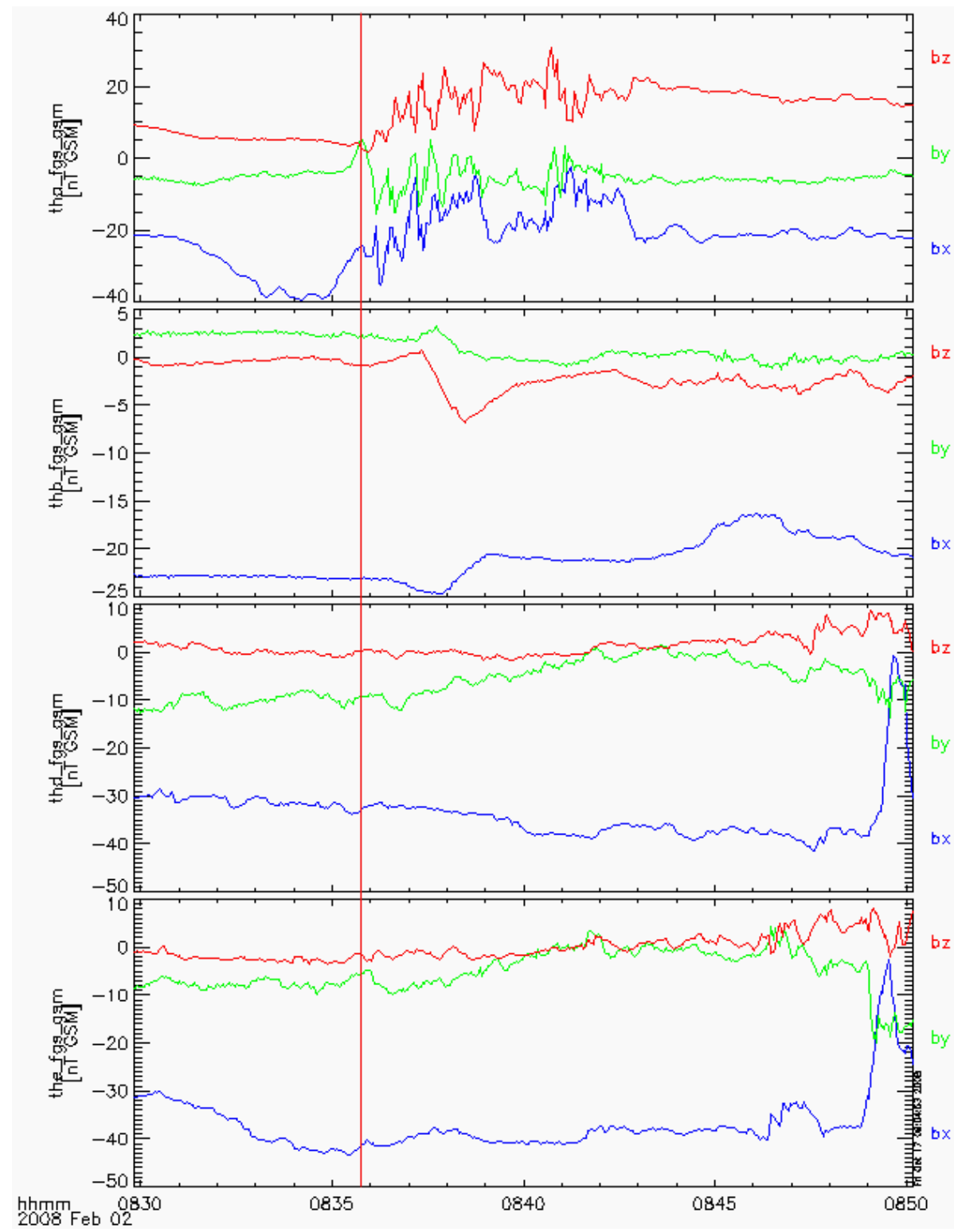

Fig. 18. THEMIS A, B, D, and E magnetometer plots. The ground onset of the expansion phase is illustrated with red vertical straight line.

reconciled with the mapping to the relatively low latitude of the auroral onset region. If the visible auroral onset region and the 11 to $17.6 R_{E}$ locations in the plasma sheet were correlated then we would have to assume substantial field line stretching in the substorm growth phase. Auroral substorm onsets are often observed in the atmosphere at 66.4 degrees magnetic latitude (dipole $L \sim 6$ ) (Frey et al., 2004) and it is conceivable that field lines from this dipole latitude would be stretched momentarily to twice or three times of their normal $\mathrm{L}$ distance distance at the end of the growth phase.

An interesting issue related to the time of origin of the magnetic impulses in relation to the ground based observation of the SPE. They seem to be in the range of a few to
$169 \mathrm{~s}$. It is interesting to note that in many cases they more or less coincide with step 2 or 3 in the substorm auroral onset process. How can we have such fast communication from such relatively large distances in the magnetosphere down to the ionosphere? All wave propagations such as Alfvén waves or ion acoustic waves would be limited in their propagation speed. At the time of dipolarization we would expect to and we often see freshly energized electrons of energy of several $\mathrm{keV}$ at the dipolarizing onset regions. The freshly accelerated electrons could arrive at the ionosphere rapidly, in a few seconds after onset. The newly injected particles would likely to be quite diffuse, quite unlike the highly structured characteristic of Alfvénic or inverted " $V$ " type aurora. These 

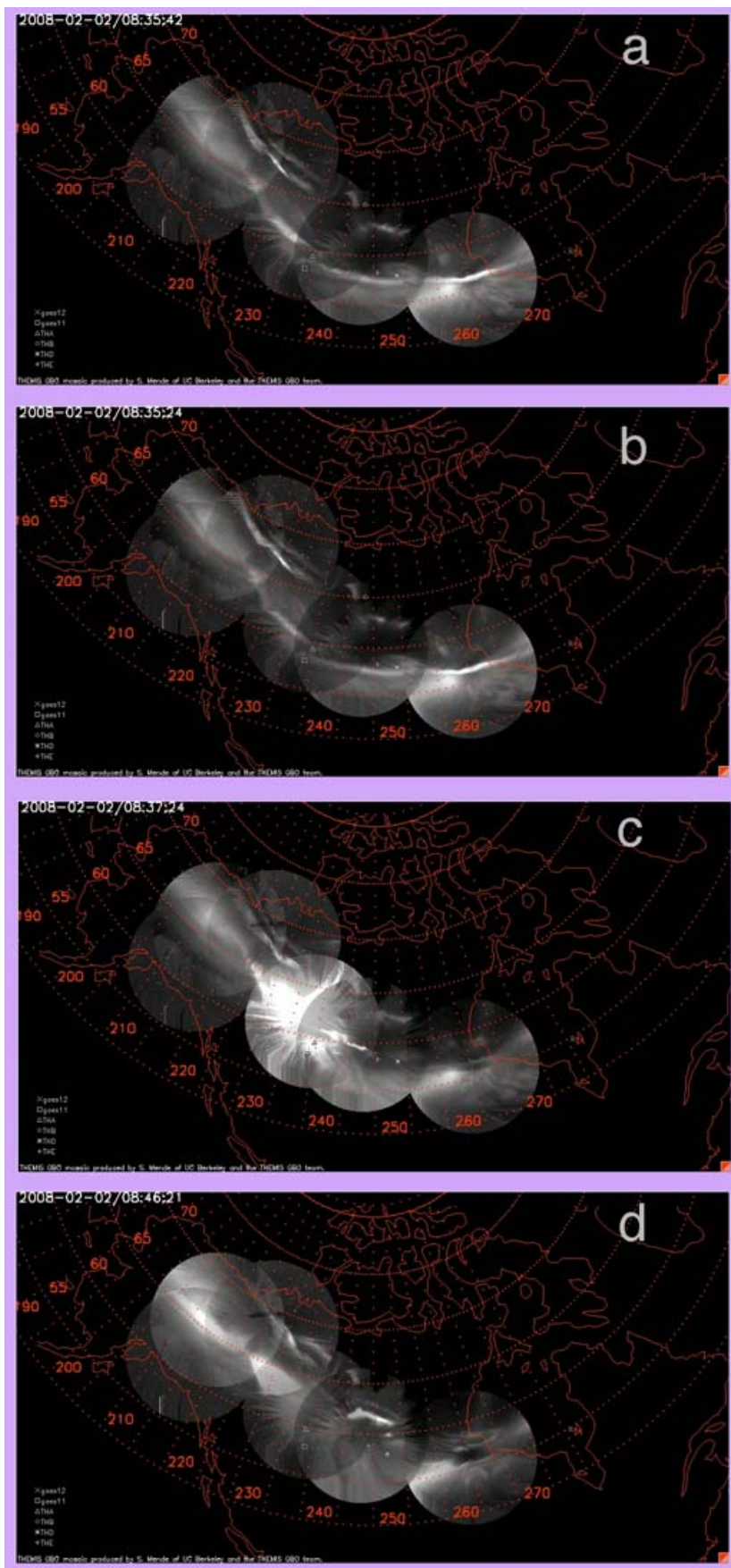

Fig. 19. Image collage representation of the $\sim 836$ event, (a) 08:35:42 ground base onset of the substorm poleward expansion phase (b) 08:35:24 magnetic signal arrival at THEMIS A (c) 08:37:24 arrival at THEMIS B (d) 08:46:21 magnetic impulse reaches satellite THEMIS E.

latter would most likely require wave propagation from the magnetosphere.

The earthward statistical limit of neutral line formation is about $17 R_{E}$ (Nagai et al., 2005) from Geotail data with the probability of occurrence rapidly rising at $20 R_{E}$ and beyond.

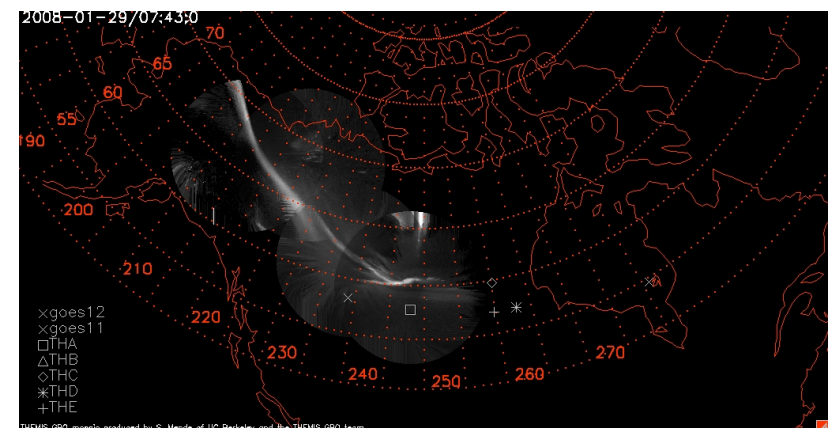

Fig. 20. The 29 January $2008 \sim 07: 41$ event showing the auroal brightening at the start of the poleward expansion (SPE) and the position of the magnetic foot prints of the THEMIS and GOES satellites.

The down-tail distance derived from our satellite onset timing is too close to the earth to be a likely place for near earth neutral line formation and corresponding re-connection

Although there are several reports in the literature that reconnection was seen to occur in individual cases nearer than $17 R_{E}$, statistical studies overwhelmingly show that reconnection closer to the earth than $20 R_{E}$ is a rare occurrence. The most recent one of these statistical studies from Geotail data shows that the statistical limit of neutral line formation is about $17 R_{E}$ (Nagai et al., 2005) with the probability of occurrence rapidly rising at $20 R_{E}$ and beyond. The down-tail distances derived from our satellite onset timing for the three independent onset events is too close to the earth to be consistent with the statistical results regarding near earth neutral line formation and corresponding re-connection.

\section{Summary}

The unprecedented coverage coupled with high temporal and spatial resolution of the THEMIS GBO station network allowed us to determine the various stages of the global scale developments of the optical aurora at substorm onsets. We used the mosaic collages of images taken during a substorm that occurred at $\sim 07: 40$ to demonstrate how to locate and time the various auroral configuration and intensity changes that signify stages of substorm onset. At the start of the Substorm Poleward Expansion (SPE) the intensity of aurora increases most rapidly and this occurrence can be timed most accurately. The plot of the magnetic x component at the station nearest to the onset is quite similar to the plot of the total auroral light intensity at the nearest station signifying the onset of the electrojet current associated with the auroral current wedge (McPheron et al, 1973). Because of the needed accuracy in the timing to accurately predict locations in the magnetosphere we advocate the use this point in time as the reference to the auroral substorm onset. The physical significance of this starting point is that it signifies the beginning of 
the large scale substorm energy dissipation in the ionosphere due to particle precipitation and auroral electrojet currents. Usually there are several distinct events preceding the SPE for example the gradual dimming of the pre-existing equatorward arc occurring several minutes prior to SPE, the relatively fast increase in the pre-existing arc intensity just a few minutes prior to SPE, and the appearance of a new dynamic rayed arc feature usually on the poleward side of the pre-existing arc. All of these pre-cursor features demonstrate that the magnetospheric region where the substorm onset energization starts must be closely coupled to the ionosphere where these precursor events occur.

Having determined the location and time of the ground based onset of the expansive phase we attempted to independently establish the location and time of the onset in the magnetosphere using only the satellite data. We used the magnetic signature of the substorm as seen by those THEMIS satellites whose magnetic foot point was closest to the onset meridian as seen in the aurora. We made the assumption that the substorm occurred between two THEMIS satellites and it propagated in and out along the magnetic tail along the $\mathrm{x}$ dimension. From the time of arrival at two satellites we calculated the location of the starting point and the time of start. In these calculations we used a model of propagation velocity of magnetic impulses as calculated from the magnetic field models and a constant plasma density and energy that was measured. The calculations allowed for a variable propagation velocity but this made very little difference from a simple linear calculation. In the valid cases where the time difference between the two satellites detecting the onset signature was less than the propagation time of a magnetic impulse traveling from one satellite to the other we found that the onset location was in a range greater than 11 and less than $17.6 R_{E}$. The time of onset in the magnetosphere preceded the ground onset SPE between about 0 to $169 \mathrm{~s}$. This means that the step 2 and 3 precursor events can be occurring simultaneously with the initiation of the magnetic impulse in the magnetosphere.

In several cases it was clear from GBO observations that the local time (azimuthal) propagation of the substorm aurora took several minutes to reach the meridian of a THEMIS satellite and therefore the timing from that satellite was highly unreliable unless there was a way to correct for the azimuthal propagation delay. We made several attempts at such corrections but had no success in developing a consistent model. It should be noted that in most such cases the difference in time between the two satellites seeing the onset signature was larger than the time of propagation of a magnetic impulse from one satellite to the other. This inconsistency could signify that the onset occurred outside of the $\mathrm{x}$ dimension region of the two satellites but it is more likely that we do not understand how to correct for delays due to substorms propagating azimuthally or in local time.
Acknowledgements. Topical Editor R. Nakamura thanks two anonymous referees for their help in evaluating this paper.

\section{References}

Akasofu, S.-I.: The development of the auroral substorm, Planet. Space Sci., 12, 273-282, 1964.

Angelopoulos, V.: The THEMIS mission, Space Sci. Rev., 141, 534, doi:10.1007/s11214-008-9336-1, 2008a.

Angelopoulos, V., McFadden, J. P., Larson, D., Carlson, C. W., Mende, S. B., Frey, H., Phan, T., Sibeck, D. G., Glassmeier, K.H., Auster, U., Donovan, E., Mann, I. R., Rae, I. J., Russell, C. T., Runov, A., Zhou, X.-Z., and Kepko, L.: Tail Reconnection Triggering Substorm, Science, 321(5891), 931-935, 2008 b.

Baker, D. N., Pulkkinen, T., Angelopoulos, V., Baumjohann, W., and McPherron, R.: Neutral line model of substorms: Past results and present view, J. Geophys. Res., 101, 12975-13010, 1996.

Donovan, E., Mende, S. B., Jackel, B., et al.: The THEMIS allsky imaging array-system design and initial results from the prototype imager, J. Atmos. Sol. Terr. Phys., 68, 1472-1487, doi:10.1016/j.jastp.2005.03.027, 2006.

Donovan, E., Liu, W., Liang, J., Spanswick, E., et al.: Simultaneous THEMIS in situ and auroral observations of a small substorm, Geophys. Res. Lett., 35, L17S18, doi:10.1029/2008GL033794, 2008.

Frey, H. U., Mende, S. B., Angelopoulos, V., and Donovan, E. F.: Substorm onset observations by IMAGE-FUV, J. Geophys. Res., 109, A10304, doi:10.1029/2004JA010607, 2004.

Kiehas, S. A., Semenov, V. S., Kubyshkina, M. V., et al.: First application of a Petschek-type reconnection model with time-varying reconnection rate to THEMIS observations. J. Geophys. Res., 114, A00C20, doi:10.1029/2008JA013528, 2009.

Lessard, M. R., Lund, E. J., Mouikis, C., Engebretson, M. J., and Mende, S. B.: Pi1B pulsations and Alfvénic aurora at substorm onset, American Geophysical Union, Fall Meeting 2006, abstract \#SM43D-01, 2006.

Lui, A. T. Y., Chang, C.-L., Mankofsky, A., Wong, H.-K., and Winske, D.: A Cross-Field Current Instability for Substorm Expansions, J. Geophys. Res., 96(A7), 11389-11401, 1991.

Lui, A. T. Y., Angelopoulos, V., LeContel, O., et al.: Determination of the substorm initiation region from a major conjunction interval of THEMIS satellites, J. Geophys. Res., 113, A00C04, doi:10.1029/2008JA013424, 2008.

Lyons, L. R., Voronkov, I. O., Donovan, E. F., and Zesta, E.: Relation of substorm breakup arc to other growth-phase auroral arcs, J. Geophys. Res., 107(A11), 1390, doi:10.1029/2002JA009317, 2002.

McPherron, R. L., Russell, C. T., and Aubry, M.: Satellite studies of magnetospheric substorms on August 15, 1978, 9, Phenomenological model for substorms, J. Geophys. Res., 78, 3131-3149, 1973.

Mende, S. B., Carlson, C. W., Frey, H. U., Peticolas, L. M., and Østgaard, N.: FAST and IMAGE-FUV observations of a substorm onset, J. Geophys. Res., 108(A9), 1344, doi:10.1029/2002JA009787, 2003.

Mende, S. B., Harris, S. E., Frey, H. U., et al.: The THEMIS array of ground based observatories for the study of auroral substorms, Space Sci. Rev., 141, 357-387, doi:10.1007/s11214-008-9380-x, 2008. 
Mende, S. B., Angelopoulos, V., Frey, H. U., Harris, S., Donovan, E., Jackel, B., Syrjaesuo, M., Russell, C. T., and Mann, I.: Determination of sub-storm onset timing and location using the THEMIS ground based observatories, Geophys. Res. Lett., 34, L17108, doi:10.1029/2007GL030850, 2007.

Moore, T. E., Gallagher, D. L., Horwitz, J. L., and Comfort, R. H.: MHD wave breaking in the outer plasmasphere, Gephys. Res. Lett., 14, 1007-1010, 1987.

Nagai, T., Fujimoto, M., Nakamura, R., Baumjohann, W., Ieda, A., Shinohara, I., Machida, S., Saito, Y., and Mukai, T.: Solar wind control of the radial distance of the magnetic reconnection site in the magnetotail, J. Geophys. Res., 110, A09208, doi:10.1029/2005JA011207, 2005.
Pellinen, R. J. and Heikkila, W. J.: Observations of auroral fading before breakup, J. Geophys. Res., 83, 4207-4217, 1978.

Roux, A., Perraut, S., Robert, P., Morane, A., Pedersen, A., Korth, A., Kremser, G., Aparicio, B., Rodgers, D., and Pellinen, R.: Plasma sheet instability related to the westward traveling surge, J. Geophys. Res., 96, 17697-17714, 1991. 